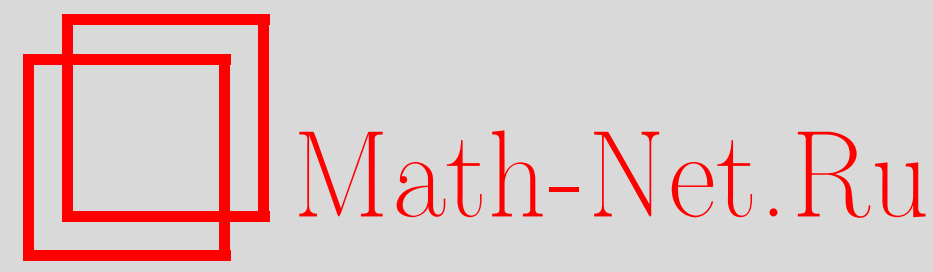

В. И. Седенко, Классическая разрешимость начально-краевой задачи нелинейной теории колебаний пологих оболочек, Изв. РАН. Сер. матем., 1996, том 60, выпуск $5,157-190$

DOI: https://doi.org/10.4213/im90

Использование Общероссийского математического портала Math-Net.Ru подразумевает, что вы прочитали и согласны с пользовательским соглашением

http://www . mathnet.ru/rus/agreement

Параметры загрузки:

IP : 54.92 .164 .108

26 апреля 2023 г., 14:04:09 
УДК 517.944

\author{
В. И. Седенко
}

\title{
Классическая разрешимость начально-краевой задачи нелинейной теории колебаний пологих оболочек
}

\footnotetext{
Доказываются теоремы о глобальной по времени априорной оценке решений, о классической разрешимости и о гладкости решений системы уравнений Маргерра-Власова без учета инерции продольных перемещений с краевыми условиями жесткого защемления края оболочки.

Библиография: 8 наименований.
}

Результаты о разрешимости в целом по времени рассматриваемой начальнокраевой задачи до настояшей работы касались лишь обобшенных решений. В 1957 г. И.И. Ворович в [1] доказал существование обобщенных решений. А недавно в [2], [3] была доказана единственность обобщенных решений.

Среди результатов настояшей работы базовым фактом является наличие априорной оценки. Выводу априорной оценки посвящены $\S 2-8$. Предлагается схема исследования, позволяющая в итоге вывести нелинейное дифференциальное неравенство, интегрирование которого и дает центральную априорную оценку. Суть схемы состоит в оценке получающихся нелинейных величин как с помощью растущих, так и с помощью убывающих по некоторому параметру слагаемых. Подходящий выбор параметра позволяет осушествить интегрирование дифференщиального неравенства. По существу речь идет о преодолении трудностей, связанных с отсутствием вложения $H_{2}^{1}(\Omega) \subset L_{\infty}(\Omega)$ в двумерном случае. В техническом плане следует отметить использование операторов сглаживания в непривычной ситуации. Теорема 2 при наличии априорной оценки доказывается, по существу, автоматически (см. $\S 9$ ). Что касается теоремы 3 , то для ее доказательства также приходится получать оценку. Этот процесс не столь сложен, поскольку мы уже обладаем достаточно сильными оценками (1.18)-(1.20). Укажем также, что частным случаем полученного результата являются теоремы классической разрешимости и гладкости решений начально-краевой задачи для уравнений колебаний пластины, существование обобщенных решений которой было доказано в [4].

\section{§1. Начально-краевая задача. Формулировки результатов}

Предположим, что оболочка проектируется на плоскую ограниченную область $\Omega$ с гранищей Г. Рассмотрим систему уравнений

$$
\begin{aligned}
\rho h w_{t t}+\mathcal{D} \nabla^{4} w= & Z+\left(N_{1} w_{x_{1}}\right)_{x_{1}}+\left(N_{12} w_{x_{1}}\right)_{x_{2}} \\
& +\left(N_{2} w_{x_{2}}\right)_{x_{2}}+\left(N_{12} w_{x_{2}}\right)_{x_{1}}-N_{1} k_{1}-N_{2} k_{2},
\end{aligned}
$$

$$
\text { (C) В.И. СЕдЕнко }
$$




$$
\begin{gathered}
N_{1}=\frac{E h}{1-\mu^{2}}\left(\varepsilon_{1}+\mu \varepsilon_{2}\right), \quad N_{2}=\frac{E h}{1-\mu^{2}}\left(\varepsilon_{2}+\mu \varepsilon_{1}\right), \quad N_{12}=\frac{E h}{2(1+\mu)} \varepsilon_{12} \\
\varepsilon_{1}=u_{x_{1}}+k_{1} w+\frac{1}{2} w_{x_{1}}^{2}, \quad \varepsilon_{2}=v_{x_{2}}+k_{2} w+\frac{1}{2} w_{x_{2}}^{2} \\
\varepsilon_{12}=u_{x_{2}}+v_{x_{1}}+w_{x_{1}} w_{x_{2}}, \\
\Delta u+\frac{1+\mu}{1-\mu} \theta_{x_{1}}=-\frac{2}{1-\mu}\left[\left(k_{1} w\right)_{x_{1}}+w_{x_{1}} w_{x_{1} x_{1}}+\mu\left(k_{2} w\right)_{x_{1}}+\mu w_{x_{1}} w_{x_{1} x_{2}}\right] \\
-w_{x_{2}} w_{x_{1} x_{2}}-w_{x_{1}} w_{x_{2} x_{2}}-X \\
\Delta v+\frac{2}{1+\mu} \theta_{x_{2}}=-\frac{2}{1-\mu}\left[\left(k_{2} w\right)_{x_{2}}+w_{x_{2}} w_{x_{2} x_{2}}+\mu\left(k_{1} w\right)_{x_{2}}+\mu w_{x_{1}} w_{x_{1} x_{2}}\right] \\
-w_{x_{1}} w_{x_{1} x_{2}}-w_{x_{2}} w_{x_{1} x_{2}}-Y
\end{gathered}
$$

где $\theta=u_{x_{1}}+v_{x_{2}}$, с краевыми условиями

$$
\begin{gathered}
\left.w\right|_{\Gamma}=\left.\frac{\partial w}{\partial n}\right|_{\Gamma}=0, \\
\left.u\right|_{\Gamma}=\left.v\right|_{\Gamma}=0
\end{gathered}
$$

и начальными условиями

$$
\begin{gathered}
w(x, 0)=w_{0}(x), \quad x \in \Omega, \\
w_{t}(x, 0)=w_{1}(x), \quad x \in \Omega .
\end{gathered}
$$

В уравнениях (1.1)-(1.9) $u, v$ - продольные перемешения, $w$ - поперечное перемещение точек срединной поверхности, $\mathcal{D}$ - изгибная жесткость оболочки, $E, \mu-$ упругие постоянные, $h$ - высота оболочки, $N_{1}, N_{2}, N_{12}$ - продольные усилия в оболочке, $\varepsilon_{1}, \varepsilon_{2}, \varepsilon_{12}$ - характеристики деформации срединной поверхности оболочки, $k_{1}, k_{2}$ - кривизны оболочки, которые считаем непрерывно дифференцируемыми в $\Omega, X, Y, Z$ - составляющие внешних сил, действующих на оболочку.

Предполагается, что далее массовая плотность и линейные размеры измеряются в таких единицах, что имеют место соотношения

$$
\rho h=1, \quad \frac{2 \rho}{E}(1+\mu)=1,
$$

где $\rho$ - массовая плотность оболочки.

Относительно начально-краевой задачи (1.1)-(1.9) см. [1, с. 759-761].

Мы будем искать решения $w, u, v$ начально-краевой задачи (1.1)-(1.9) на отрезке времени $\left[0, t_{f}\right]$, где $t_{f}-$ конечная, но никакими условиями не ограниченная величина. 
Через $H_{p}^{l}(\Omega), l=0,1, \ldots, 1 \leqslant p \leqslant \infty$, обозначим банахово пространство функций на $\Omega$, являющееся пополнением множества бесконечно дифференцируемых на $\Omega$ функций по следующей норме:

$$
\|u\|_{H_{p}^{l}(\Omega)}=\sum_{|r| \leqslant l}\left\|D^{r} u\right\|_{L_{p(\Omega)}}
$$

где $r=\left(r_{1}, r_{2}\right),|r|=r_{1}+r_{2}, D^{r} u$ - частная производная функции $u$ порядка $r$. Далее, через $\stackrel{\circ}{H}_{p}^{l}(\Omega), l=0,1, \ldots, 1 \leqslant p \leqslant \infty$, обозначим банахово пространство функций на $\Omega$, являющееся пополнением множества бесконечно дифференцируемых финитных на $\Omega$ функций по следующей норме:

$$
\|u\|_{\stackrel{\circ}{p}_{p}^{l}(\Omega)}=\sum_{|r|=l}\left\|D^{r} u\right\|_{L_{p}(\Omega)}
$$

Далее нам придется получать сильные априорные оценки решений (1.1)-(1.9). При этом, разумеется, мы будем использовать уже известные оценки решений этой задачи. Мы приведем здесь основные из них с тем, чтобы удобнее было на них ссылаться. Дело в том, что решения (1.1)-(1.9) удовлетворяют энергетическому соотношению, в силу которого функционал $\Phi$ потенциальной энергии изгиба и растяжения оболочки сушественно ограничен на отрезке времени $\left[0, t_{f}\right]$ в случае, когда $w_{0} \in \stackrel{\circ}{H}_{2}^{2}(\Omega), w_{1} \in L_{2}(\Omega), X, Y, Z \in L_{2}\left(\Omega \times\left[0, t_{f}\right]\right)$.

Здесь

$$
\Phi=\frac{1}{2}\|w\|_{H_{2}^{2}(\Omega)}^{2}+\frac{E h}{2\left(1-\mu^{2}\right)} \int_{\Omega}\left[\varepsilon_{1}^{2}+\varepsilon_{2}^{2}+2 \mu \varepsilon_{1} \varepsilon_{2}+\frac{1}{2}(1-\mu) \varepsilon_{12}^{2}\right] d x .
$$

Таким образом, имеем

$$
\underset{t \in\left[0, t_{f}\right]}{\operatorname{vraimax}} \Phi(t) \leqslant B_{1}
$$

(см. $[1,(1.36)])$. Из (1.10) следует оценка

$$
\underset{t \in\left[0, t_{f}\right]}{\operatorname{vrai} \max }\|w(x, t)\|_{H_{2}^{2}(\Omega)} \leqslant B_{2}
$$

Кроме того, имеет место оценка

$$
\underset{t \in\left[0, t_{f}\right]}{\operatorname{vraimax}}\left\|w_{t}(x, t)\right\|_{\stackrel{\circ}{2}_{2}^{1}(\Omega)} \leqslant B_{3} .
$$

Она следует из $[1,(1.34)]$. Константы $B_{1}, B_{2}, B_{3}$ зависят лишь от нормы $w_{0}$ в $\stackrel{\circ}{H}_{2}^{2}(\Omega)$, от норм $w_{1}, X, Y, Z$ в $L_{2}\left(\Omega \times\left[0, t_{f}\right]\right)$, от величин коэффиициентов в $(1.1)-(1.9)$ 
и от области $\Omega$. Оценки $(1.11),(1.12)$ основные. Из них с помошью теорем вложения следуют такие полезные для дальнейшего оценки:

$$
\begin{aligned}
& \underset{t \in\left[0, t_{f}\right]}{\operatorname{vraimax}}\|w(x, t)\|_{L_{\infty}(\Omega)} \leqslant B_{4}, \\
& \underset{t \in\left[0, t_{f}\right]}{\operatorname{vrai} \max }\|w(x, t)\|_{H_{p}^{1}(\Omega)} \leqslant B_{5}^{p} .
\end{aligned}
$$

Константа $B_{4}$ зависит от тех же величин, что и константы $B_{i}, i=1,2,3$, а $B_{5}^{p}$ зависит еще дополнительно от $p$.

Теперь укажем оценки функций $u$ и $v$. Поскольку дифференциальные операторы левых частей (1.4) и (1.5) составляют коэрцитивную систему, так как удовлетворяют условию теоремы 11.3 из [5, с. 159], то для всех $p, 1<p<2$, имеют место следуюшие оценки:

$$
\underset{t \in\left[0, t_{f}\right]}{\operatorname{vraimax}}\left[\sum_{i, j=1}^{2}\left(\left\|u_{x_{i} x_{j}}(x, t)\right\|_{L_{p}(\Omega)}+\left\|v_{x_{i} x_{j}}(x, t)\right\|_{L_{p}(\Omega)}\right)\right] \leqslant B_{6}^{p} .
$$

Эта оценка получается с учетом вида правых частей (1.4) и (1.5) и оценкой их с помощью (1.11) и (1.14). Константа $B_{6}^{p}$ зависит от тех же величин, что и константа $B_{5}^{p}$.

Из (1.15) и (1.7) для всех $p, 1 \leqslant p<\infty$, следует оценка

$$
\underset{t \in\left[0, t_{f}\right]}{\operatorname{vraimax}}\left(\|u(x, t)\|_{H_{p}^{1}(\Omega)}+\|v(x, t)\|_{H_{p}^{1}(\Omega)}\right) \leqslant B_{7}^{p} .
$$

Константа $B_{7}^{p}$ зависит от тех же величин, что и константа $B_{5}^{p}$.

Теперь мы изложим основные результаты настоящей работы. Основополагаюшим фактом для результатов о разрешимости является наличие достаточно сильной априорной оценки. Приведем формулировку теоремы об априорной оценке.

Teopema 1.1. Пусть $\Gamma \in C_{a}, \quad k_{1}, k_{2} \in C^{1}(\Omega), \quad w_{0}, w_{1} \in C^{4}(\Omega), \quad X, Y, Z \in$ $C^{2,1}\left(\Omega \times\left[0, t_{f}\right]\right)$. Кроме того, пусть для самого решения начально-краевой задачи (1.1)-(1.9) $w_{t t t}: \nabla^{4} w \in C\left(\Omega \times\left[0, t_{f}\right]\right)$.

Тогда имеют место следующие оценки:

$$
\begin{aligned}
& \max _{t \in\left[0, t_{f}\right]}\left(\left\|w_{t t}(x, t)\right\|_{L_{2}(\Omega)}+\left\|\Delta w_{t}(x, t)\right\|_{L_{2}(\Omega)}\right) \leqslant K_{1}, \\
& \max _{t \in\left[0, t_{f}\right]}\left\|\nabla^{4} w(x, t)\right\|_{L_{2}(\Omega)} \leqslant K_{2}, \\
& \max _{t \in\left[0, t_{f}\right]}\left(\|u(x, t)\|_{H_{2}^{4}(\Omega)}+\|v(x, t)\|_{H_{2}^{4}(\Omega)}\right) \leqslant K_{3},
\end{aligned}
$$


где константы $K_{1}, K_{2}, K_{3}$ зависят лишь от констант $B_{i}, i=1, \ldots, 7$, от величины нормы $w_{0}$ в $H_{2}^{4}(\Omega)$, от величины нормы $w_{1}$ в $L_{2}(\Omega)$, от величин

$$
\begin{aligned}
& \max _{t \in\left[0, t_{f}\right]}\left(\|X(x, t)\|_{H_{2}^{2}(\Omega)}+\left\|X_{t}(x, t)\right\|_{L_{2}(\Omega)}\right), \\
& \max _{t \in\left[0, t_{f}\right]}\left(\|Y(x, t)\|_{H_{2}^{2}(\Omega)}+\left\|Y_{t}(x, t)\right\|_{L_{2}(\Omega)}\right), \\
& \max _{t \in\left[0, t_{f}\right]}\left(\|Z(x, t)\|_{L_{2}(\Omega)}+\left\|Z_{t}(x, t)\right\|_{L_{2}(\Omega)}\right)
\end{aligned}
$$

и от области $\Omega$.

Класс $C_{a}$ гладкости границы $\Gamma$ области $\Omega$ будет описан по завершению доказательства теоремы 1 в $\S 8$. Он не является сколько-нибудь оригинальным, определяясь лишь условиями, которые достаточны для выполнения некоторых неравенств коэрцитивности. Все четырежды непрерывно дифференцируемые границы заведомо входят в этот класс.

Теперь сформулируем теоремы о разрешимости.

Teopema 1.2. Пусть $\Gamma \in C_{a}, \quad k_{1}, k_{2} \in C^{1}(\Omega), \quad w_{0} \in \stackrel{\circ}{H}_{2}^{2}(\Omega) \cap H_{2}^{4}(\Omega), \quad w_{1} \in$ $\stackrel{\circ}{H}_{2}^{2}(\Omega)$. Пусть, кроме того,

$$
\begin{aligned}
& \underset{t \in\left[0, t_{f}\right]}{\operatorname{vraimax}}\left(\|X(x, t)\|_{H_{2}^{2}(\Omega)}+\left\|X_{t}(x, t)\right\|_{L_{2}(\Omega)}\right)<\infty, \\
& \underset{t \in\left[0, t_{f}\right]}{\operatorname{vraimax}}\left(\|Y(x, t)\|_{H_{2}^{2}(\Omega)}+\left\|Y_{t}(x, t)\right\|_{L_{2}(\Omega)}\right)<\infty, \\
& \underset{t \in\left[0, t_{f}\right]}{\operatorname{vraimax}}\left(\|Z(x, t)\|_{H_{2}^{2}(\Omega)}+\left\|Z_{t}(x, t)\right\|_{L_{2}(\Omega)}\right)<\infty .
\end{aligned}
$$

Тогда существует функщия $w(x, t)$ на $\Omega \times\left[0, t_{f}\right]$, для которой выполняются следующие оченки:

$$
\begin{aligned}
& \underset{t \in\left[0, t_{f}\right]}{\operatorname{vraimax}}\left(\left\|w_{t t}(x, t)\right\|_{L_{2}(\Omega)}+\left\|\Delta w_{t}(x, t)\right\|_{L_{2}(\Omega)}\right) \leqslant K_{1}, \\
& \underset{t \in\left[0, t_{f}\right]}{\operatorname{vraimax}}\left\|\nabla^{4} w(x, t)\right\|_{L_{2}(\Omega)} \leqslant K_{2}, \\
& \underset{t \in\left[0, t_{f}\right]}{\operatorname{vraimax}}\left(\|u(x, t)\|_{H_{2}^{4}(\Omega)}+\|v(x, t)\|_{H_{2}^{4}(\Omega)}\right) \leqslant K_{3},
\end{aligned}
$$

причем $w, u, v$ удовлетворяют начально-краевой задаче (1.1)-(1.9) почти всюду .

Наконец, сформулируем теорему о гладкости решений начально-краевой задачи (1.1)-(1.9). 
TEOpEMA 1.3. Пусть $r \in \mathbb{Z}^{+}, \quad \Gamma \in C_{a}^{r}, \quad k_{1}, k_{2} \in H_{2}^{2 r-2}(\Omega), \quad w_{0} \in \stackrel{\circ}{H_{2}^{2}}(\Omega) \cap$ $H_{2}^{4 r}(\Omega), \quad w_{1} \in \stackrel{\circ}{H}_{2}^{2}(\Omega) \cap H_{2}^{4 r-2}(\Omega)$. Пусть в условиях теоремь 2

$$
\begin{aligned}
& \max _{p+q=r-1} \operatorname{vraimax}_{t \in\left[0, t_{f}\right]}\left\|\frac{\partial^{p}}{\partial t^{p}} X(x, t)\right\|_{H_{2}^{2 q}(\Omega)}=B_{8}^{r}<\infty, \\
& \max _{p+q=r-1} \underset{t \in\left[0, t_{f}\right]}{\operatorname{vraimax} \max }\left\|\frac{\partial^{p}}{\partial t^{p}} Y(x, t)\right\|_{H_{2}^{2 q}(\Omega)}=B_{9}^{r}<\infty, \\
& \max _{p+q=r-1} \underset{t \in\left[0, t_{f}\right]}{\operatorname{vrai} \max }\left\|\frac{\partial^{p}}{\partial t^{p}} Z(x, t)\right\|_{H_{2}^{2 q}(\Omega)}=B_{10}^{r}<\infty \\
& \underset{t \in\left[0, t_{f}\right]}{\operatorname{vraimax}}\left(\left\|\frac{\partial^{r}}{\partial t^{r}} X(x, t)\right\|_{L_{2}(\Omega)}+\left\|\frac{\partial^{r}}{\partial t^{r}} Y(x, t)\right\|_{L_{2}(\Omega)}\right. \\
& \left.+\left\|\frac{\partial^{r}}{\partial t^{r}} Z(x, t)\right\|_{L_{2}(\Omega)}\right)=B_{11}^{r}<\infty .
\end{aligned}
$$

Тогда существует решение $w(x, t)$ начально-краевой задачи (1.1)-(1.9), удовлетворяющее следующим оценкам:

$$
\begin{gathered}
\max _{p+q=r+1} \underset{t \in\left[0, t_{f}\right]}{\operatorname{vraimax}}\left\|\frac{\partial^{p}}{\partial t^{p}} w(x, t)\right\|_{H_{2}^{2 q}(\Omega)} \leqslant K_{4}^{r}, \\
\max _{p+q=r+1} \operatorname{vraimax}_{t \in\left[0, t_{f}\right]}\left(\left\|\frac{\partial^{p}}{\partial t^{p}} u(x, t)\right\|_{H_{2}^{2 q}(\Omega)}+\left\|\frac{\partial^{p}}{\partial t^{p}} v(x, t)\right\|_{H_{2}^{2 q}(\Omega)}\right) \leqslant K_{5}^{r} .
\end{gathered}
$$

Константы $K_{4}^{r}, K_{5}^{r}$ зависят лишь от величин, указанных в формулировке теоремы 1.1 и от констант $B_{8}^{r}, B_{9}^{r}, B_{10}^{r}, B_{11}^{r}$.

Опять-таки объяснения относительно класса гладкости гранищы Г области $\Omega$ мы дадим после доказательства теоремы 1.3. Укажем лишь, что все границы гладкости $C^{2 r+2}$ входят в $C_{a}^{r}$.

\section{§ 2. Начало доказательства теоремы 1.1}

В условиях теоремы 1.1 в $\S 2-8$ мы произведем оценку величины

$$
\xi(t)=\left\|w_{t t}(x, t)\right\|_{L_{2}(\Omega)}^{2}+\mathcal{D}\left\|\Delta w_{t}(x, t)\right\|_{L_{2}(\Omega)}^{2} .
$$

Для этого сначала продифференцируем уравнение (1.1) по $t$. Мы получим следующее уравнение:

$$
\begin{gathered}
w_{t t t}+\mathcal{D} \nabla^{4} w_{t}=Z_{t}+\frac{\partial}{\partial x_{1}}\left(N_{1} w_{x_{1}}\right)_{t}+\frac{\partial}{\partial x_{2}}\left(N_{12} w_{x_{1}}\right)_{t}+\frac{\partial}{\partial x_{2}}\left(N_{2} w_{x_{2}}\right)_{t} \\
+\frac{\partial}{\partial x_{1}}\left(N_{12} w_{x_{2}}\right)_{t}-k_{1} N_{1 t}-k_{2} N_{2 t}
\end{gathered}
$$


Выполняются следующие краевые условия:

$$
\left.w_{t}\right|_{\Gamma}=\left.\frac{\partial w_{t}}{\partial n}\right|_{\Gamma}=0
$$

Умножим теперь $(2.1)$ на $w_{t t}(x, t)$ скалярно в $L_{2}(\Omega)$. Интегрируя по частям по $x$, с учетом (2.2) получим неравенство

$$
\xi_{t}(t) \leqslant \sigma_{1} \sum_{l=1}^{14} A_{l}(t)
$$

где константа $\sigma_{1}$ зависит лишь от неизменяюшихся данных (коэффициентов) задачи (1.1)-(1.9). Здесь

$$
\begin{aligned}
& A_{1}(t)=\left(Z_{t}(x, t), w_{t t}(x, t)\right)_{L_{2}(\Omega)}, \\
& A_{2}(t)=\sum_{i=1}^{2}\left\|w_{t}(x, t) w_{x_{i}}(x, t) w_{t t}(x, t)\right\|_{L_{1}(\Omega)}, \\
& A_{3}(t)=\sum_{i=1}^{2}\left\|w(x, t) w_{x_{i} t}(x, t) w_{t t}(x, t)\right\|_{L_{1}(\Omega)}, \\
& A_{4}(t)=\sum_{i, j=1}^{2}\left\|w_{x_{i}}(x, t) w_{x_{j} t}(x, t) w_{t t}(x, t)\right\|_{L_{1}(\Omega)}, \\
& A_{5}(t)=\sum_{i, j=1}^{2}\left\|w_{x_{i} x_{j}}(x, t) w_{t}(x, t) w_{t t}(x, t)\right\|_{L_{1}(\Omega)}, \\
& A_{6}(t)=\sum_{i, j=1}^{2}\left\|w_{x_{i} x_{j} t}(x, t) w(x, t) w_{t t}(x, t)\right\|_{L_{1}(\Omega)}, \\
& A_{7}(t)=\left\|w_{t}(x, t) w_{t t}(x, t)\right\|_{L_{1}(\Omega)}, \\
& A_{8}(t)=\sum_{i, j, r, s=1}^{2}\left\|w_{x_{i}}(x, t) w_{x_{j}}(x, t) w_{x_{r} x_{s}}(x, t) w_{t t}(x, t)\right\|_{L_{1}(\Omega)}, \\
& A_{10}(t)=\sum_{i=1}^{2}\left(\left\|u_{x_{i} t}(x, t) w_{t t}(x, t)\right\|_{L_{1}(\Omega)}+\left\|v_{x_{i} t}(x, t) w_{t t}(x, t)\right\|_{L_{1}(\Omega)}\right), \\
& \sum_{i, j, r, s=1}\left\|w_{x_{i}}(x, t) w_{x_{j} t}(x, t) w_{x_{r} x_{s}}(x, t) w_{t t}(x, t)\right\|_{L_{1}(\Omega)},
\end{aligned}
$$




$$
\begin{aligned}
A_{11}(t)= & \sum_{i, j, r=1}^{2}\left(\left\|w_{x_{i} t}(x, t) u_{x_{j} x_{r}}(x, t) w_{t t}(x, t)\right\|_{L_{1}(\Omega)}\right. \\
& \left.+\left\|w_{x_{i} t}(x, t) v_{x_{j} x_{r}}(x, t) w_{t t}(x, t)\right\|_{L_{1}(\Omega)}\right), \\
A_{12}(t)= & \sum_{i, j, r=1}^{2}\left(\left\|w_{x_{i} x_{j} t}(x, t) u_{x_{r}}(x, t) w_{t t}(x, t)\right\|_{L_{1}(\Omega)}\right. \\
& \left.+\left\|w_{x_{i} x_{j} t}(x, t) v_{x_{r}}(x, t) w_{t t}(x, t)\right\|_{L_{1}(\Omega)}\right), \\
A_{13}(t)= & \sum_{i, j, r=1}^{2}\left(\left\|w_{x_{i}}(x, t) u_{x_{j} x_{r} t}(x, t) w_{t t}(x, t)\right\|_{L_{1}(\Omega)}\right. \\
& \left.+\left\|w_{x_{i}}(x, t) v_{x_{j} x_{r} t}(x, t) w_{t t}(x, t)\right\|_{L_{1}(\Omega)}\right), \\
A_{14}(t)= & \sum_{i, j, r=1}^{2}\left(\left\|w_{x_{i} x_{j}}(x, t) u_{x_{r} t}(x, t) w_{t t}(x, t)\right\|_{L_{1}(\Omega)}\right. \\
& \left.+\left\|w_{x_{i} x_{j}}(x, t) v_{x_{r} t}(x, t) w_{t t}(x, t)\right\|_{L_{1}(\Omega)}\right) .
\end{aligned}
$$

Теперь мы можем оценить все величины $A_{k}(t), k \in \mathbb{Z}^{+}, 1 \leqslant k \leqslant 14$. Часть из них оценивается просто. Эти простые оценки мы приведем здесь. Используя неравенства Шварца и Юнга, имеем

$$
\begin{aligned}
A_{1}(t) & \leqslant\left\|Z_{t}(x, t)\right\|_{L_{2}(\Omega)}\left\|w_{t t}(x, t)\right\|_{L_{2}(\Omega)} \\
& \leqslant\left\|w_{t t}(x, t)\right\|_{L_{2}(\Omega)}^{2}+\left\|Z_{t}(x, t)\right\|_{L_{2}(\Omega)}^{2} \leqslant \xi(t)+\left\|Z_{t}(x, t)\right\|_{L_{2}(\Omega)}^{2} .
\end{aligned}
$$

Далее, используя неравенство Гёльдера и ограниченное вложение $H_{2}^{2}(\Omega)$ в $L_{\infty}(\Omega)$, с учетом (1.6), (1.11) получаем

$$
\begin{aligned}
A_{2}(t) & \leqslant \sigma_{2}\left\|w_{t}(x, t)\right\|_{L_{\infty}(\Omega)}\|w(x, t)\|_{H_{2}^{1}(\Omega)}\left\|w_{t t}(x, t)\right\|_{L_{2}(\Omega)} \\
& \leqslant \sigma_{3}\left\|w_{t}(x, t)\right\|_{\stackrel{O}{2}_{2}^{2}(\Omega)}\left\|w_{t t}(x, t)\right\|_{L_{2}(\Omega)} \leqslant \sigma_{4} \xi(t) .
\end{aligned}
$$

Здесь константа $\sigma_{4}$ зависит лишь от константы $B_{2}$ и области $\Omega$.

Оценку величины $A_{3}(t)$ делаем аналогично оценке $A_{2}(t)$. Имеем

$$
\begin{aligned}
A_{3}(t) & \leqslant \sigma_{5}\|w(x, t)\|_{L_{\infty}(\Omega)}\left\|w_{t}(x, t)\right\|_{\stackrel{\circ}{2}_{2}^{1}(\Omega)}\left\|w_{t t}(x, t)\right\|_{L_{2}(\Omega)} \\
& \leqslant \sigma_{6}\|w(x, t)\|_{\stackrel{\circ}{2}_{2}^{2}(\Omega)}\left\|w_{t}(x, t)\right\|_{\stackrel{\circ}{1}_{2}^{1}(\Omega)}\left\|w_{t t}(x, t)\right\|_{L_{2}(\Omega)} \leqslant \sigma_{7} \xi(t) .
\end{aligned}
$$


Здесь константа $\sigma_{7}$ зависит лишь от констант $B_{2}$ и $B_{3}$ и от области $\Omega$. Используя неравенство Гёльдера и ограниченное вложение $H_{2}^{1}(\Omega)$ в $L_{4}(\Omega)$, с учетом $(1.6)$, а также (1.11) и (1.12) получаем

$$
\begin{aligned}
A_{4}(t) & \leqslant \sigma_{8}\|w(x, t)\|_{\stackrel{\circ}{H}_{4}^{1}(\Omega)}\left\|w_{t}(x, t)\right\|_{\stackrel{\circ}{4}_{4}^{1}(\Omega)}\left\|w_{t t}(x, t)\right\|_{L_{2}(\Omega)} \\
& \leqslant \sigma_{9}\|w(x, t)\|_{\stackrel{\circ}{2}_{2}^{2}(\Omega)}\left\|w_{t}(x, t)\right\|_{\stackrel{\circ}{2}_{2}^{2}(\Omega)}\left\|w_{t t}(x, t)\right\|_{L_{2}(\Omega)} \leqslant \sigma_{10} \xi(t) .
\end{aligned}
$$

Константа $\sigma_{10}$ зависит лишь от констант $B_{2}$ и $B_{3}$ и от области $\Omega$.

Величина $A_{5}(t)$ оценивается аналогично $A_{2}(t)$. Имеем

$$
\begin{aligned}
A_{5}(t) & \leqslant \sigma_{11}\left\|w_{t}(x, t)\right\|_{L_{\infty}(\Omega)}\|w(x, t)\|_{\stackrel{\circ}{2}_{2}^{2}(\Omega)}\left\|w_{t t}(x, t)\right\|_{L_{2}(\Omega)} \\
& \leqslant \sigma_{12}\left\|w_{t}(x, t)\right\|_{\stackrel{\circ}{2}_{2}^{2}(\Omega)}\left\|w_{t t}(x, t)\right\|_{L_{2}(\Omega)} \leqslant \sigma_{13} \xi(t) .
\end{aligned}
$$

Константа $\sigma_{13}$ зависит лишш от константы $B_{2}$ и от области $\Omega$.

Величина $A_{6}(t)$ оценивается аналогично $A_{3}(t)$. Имеем

$$
\begin{aligned}
A_{6}(t) & \leqslant \sigma_{14}\|w(x, t)\|_{L_{\infty}(\Omega)}\left\|w_{t}(x, t)\right\|_{\stackrel{\circ}{2}_{2}^{2}(\Omega)}\left\|w_{t t}(x, t)\right\|_{L_{2}(\Omega)} \\
& \leqslant \sigma_{15}\|w(x, t)\|_{\stackrel{\circ}{2}_{2}^{2}(\Omega)}\left\|w_{t}(x, t)\right\|_{\stackrel{\circ}{2}_{2}^{2}(\Omega)}\left\|w_{t t}(x, t)\right\|_{L_{2}(\Omega)} \leqslant \sigma_{16} \xi(t) .
\end{aligned}
$$

Константа $\sigma_{16}$ зависит лишь от константы $B_{2}$ и от области $\Omega$.

Наконец, $A_{7}(t)$ оценивается так же, как $A_{1}(t)$. Имеем

$$
\begin{aligned}
A_{7}(t) & \leqslant\left\|w_{t}(x, t)\right\|_{L_{2}(\Omega)}\left\|w_{t t}(x, t)\right\|_{L_{2}(\Omega)} \\
& \leqslant\left\|w_{t t}(x, t)\right\|_{L_{2}(\Omega)}^{2}+\left\|w_{t}(x, t)\right\|_{L_{2}(\Omega)}^{2} \leqslant \xi(t)+\left\|w_{t}(x, t)\right\|_{L_{2}(\Omega)}^{2} .
\end{aligned}
$$

Для оценки остальных величин $A_{k}(t), k \in \mathbb{Z}^{+}, 8 \leqslant k \leqslant 14$, нужна некоторая предварительная подготовка.

\section{§3. Оценки с использованием операторов сглаживания}

Без ограничения обшности будем считать, что $\Omega \subset D=\left\{x: x=\left(x_{1}, x_{2}\right)\right.$, $\left.0<x_{1}<2 \pi, 0<x_{2}<2 \pi\right\}$. Тогда, поскольку $\stackrel{\circ}{H}_{2}^{2}(\Omega) \subset H_{2}^{2}$, где $H_{2}^{2}-$ пополнение множества тригонометрических полиномов двух переменных по норме

$$
\|h\|_{H_{2}^{2}}=\|h\|_{L_{2}(D)}+\sum_{p, q=1}^{2}\left\|h_{x_{p} x_{q}}\right\|_{L_{2}(D)}
$$

имеем

$$
h(x)=\sum_{n=-\infty}^{\infty} a_{n} e^{i n x}, \quad\|h\|_{H_{2}^{2}(\Omega)}^{2}=\sum_{n=-\infty}^{\infty}|n|^{4}\left|a_{n}\right|^{2} .
$$


Для $N \in \mathbb{Z}^{+}$положим

$$
\begin{aligned}
& \left(T_{N} h\right)(x)=\sum_{n_{1}=-N}^{N} \sum_{n_{2}=-N}^{N} a_{n} e^{i n x}, \\
& \left(R_{N} h\right)(x)=h(x)-\left(T_{N} h\right)(x) .
\end{aligned}
$$

Очевидно, что

$$
\begin{gathered}
\left\|T_{N} h\right\|_{H_{2}^{j}}^{2}+\left\|R_{N} h\right\|_{H_{2}^{j}}^{2}=\|h\|_{\mathrm{H}_{2}^{j}(\Omega)}^{2}, \quad j=0,1,2, \\
\left\|R_{N} h\right\|_{L_{2}(\Omega)} \leqslant N^{-1}\|h\|_{\stackrel{\circ}{1}_{2}^{1}(\Omega)}, \\
\left\|R_{N} h\right\|_{\stackrel{\circ}{1}_{2}^{1}(\Omega)} \leqslant N^{-1}\|h\|_{\stackrel{\circ}{2}_{2}^{2}(\Omega)} .
\end{gathered}
$$

Оценки (3.2) и (3.3) могут быть в дальнейшем очень полезны. Однако их недостаточно, и нам придется осуществить некоторое развитие этих оценок. Положим $\Omega^{*}=\Omega \cup\left(\mathbb{R}^{2} \backslash D\right)$. Рассмотрим следующее банальное продолжение функций $h(x), x \in \Omega$, до функций $h^{*}(x)=h(x), x \in \Omega, h^{*}(x)=0, x \in \mathbb{R}^{2} \backslash D$. Пусть $P: H_{q}^{p}(\Omega) \rightarrow H_{q}^{p}\left(\mathbb{R}^{2}\right)$ - универсальньй оператор продолжения (см. [6, с. 214]), который, разумеется, ограничен. Положим теперь $(Q h)(x)=\left(P h^{*}\right)(x)$. Тогда для $h(x) \in \stackrel{\circ}{H}_{2}^{2}(\Omega) \cap H_{2}^{k}(\Omega), k>2$, имеем при $j=0,1,2$

$$
\begin{aligned}
\left\|\left(R_{N} Q h\right)(x)\right\|_{H_{2}^{j}(\Omega)} & \leqslant\left\|\left(R_{N} Q h\right)(x)\right\|_{H_{2}^{j}} \leqslant N^{-r}\|(Q h)(x)\|_{H_{2}^{j+r}} \\
& \leqslant d_{r} N^{-r}\|h(x)\|_{H_{2}^{j+r}(\Omega)}
\end{aligned}
$$

где константа $d_{r}$ зависит лишь от $r$ и $\Omega$.

Далее нам понадобится оценка, описанная в следующей лемме.

ЛЕмма 3.1. Для $h \in \stackrel{\circ}{H}_{2}^{1}(\Omega)$ и $N \in \mathbb{Z}^{+}, N \geqslant 2$, имеет место следующая оценка:

$$
\max _{x \in \Omega}\left|\left(T_{N} h\right)(x)\right| \leqslant B_{12}(\ln N)^{1 / 2}\|h\|_{H_{2}^{1}(\Omega)},
$$

где константа $B_{12}$ не зависит от $N$ u $h$.

ДокАЗАТЕльство. Имеем для $h(x)=\sum_{n=-\infty}^{\infty} a_{n} e^{i n x}$

$$
\begin{aligned}
\max _{x \in D}\left|\left(T_{N} h\right)(x)\right| & \leqslant \sum_{n_{1}=-N}^{N} \sum_{n_{2}=-N}^{N}\left|a_{n}\right|=\left|a_{0}\right|+\sum_{n_{1}=-N}^{N} \sum_{n_{2}=-N}^{N}|n|\left|a_{n}\right||n|^{-1} \\
& \leqslant\left|a_{0}\right|+\left(\sum_{n_{1}=-N}^{N} \sum_{n_{2}=-N}^{N}|n|^{2}\left|a_{n}\right|^{2}\right)^{1 / 2}\left(\sum_{n_{1}=-N}^{N} \sum_{n_{2}=-N}^{N}|n|^{-2}\right)^{1 / 2} \\
& \leqslant\left|a_{0}\right|+C\left\|\left(T_{N} h\right)(x)\right\|_{H_{2}^{1}}(\ln N)^{1 / 2}
\end{aligned}
$$


Кроме того, учтем, что

$$
\left|a_{0}\right|=\left|\int_{D} h(x) d x\right| \leqslant 2 \pi\|h\|_{L_{2}(\Omega)} \leqslant 2 \pi\|h\|_{\stackrel{\circ}{2}_{2}^{1}(\Omega)} .
$$

Тем самым получаем (3.5). Лемма доказана.

Нам придется также использовать еще одну оценку, которую мы излагаем в следующей лемме.

Лемма 3.2. Для $h \in H_{2}^{2} u N \in \mathbb{Z}^{+}, \quad N \geqslant 2$, имеет место следующая оченка:

$$
\max _{x \in D}\left|\left(R_{N} h\right)(x)\right| \leqslant B_{13} N^{-1 / 2}\|h\|_{H_{2}^{2}}
$$

где константа $B_{13}$ не зависит от $N$ и $h$.

ДокАЗАТЕЛЬСТво. Имеем для $h(x)=\sum_{n=-\infty}^{+\infty} a_{n} e^{i n x}$

$$
\begin{aligned}
\max _{x \in D}\left|\left(R_{N} h\right)(x)\right| & \leqslant \sum_{|n|>N}\left|a_{n}\right|=\sum_{|n|>N}|n|^{3 / 2}\left|a_{n} \| n\right|^{-3 / 2} \\
& \leqslant\left(\sum_{|n|>N}|n|^{3}\left|a_{n}\right|^{2}\right)^{1 / 2}\left(\sum_{|n|>N}|n|^{-3}\right)^{1 / 2} \leqslant B_{13} N^{-1 / 2}\|h\|_{H_{2}^{2}} .
\end{aligned}
$$

Лемма доказана.

\section{$\S 4$. Оценки $A_{8}(t)$ и $A_{9}(t)$}

Сначала получим несколько вспомогательных оценок, которые будут полезны далее.

ПРЕДЛОЖЕНИЕ 4.1. Для всех $t \in\left[0, t_{f}\right]$ имеют место следующие оченки:

$$
\begin{aligned}
\|w(x, t)\|_{H_{p}^{4}(\Omega)} & \leqslant \sigma_{17}^{p}(\xi(t)+\eta)^{1 / 2}, \quad 1<p<2, \\
\|w(x, t)\|_{H_{2}^{3}(\Omega)} & \leqslant \sigma_{18}(\xi(t)+\eta)^{1 / 2} \\
\|w(x, t)\|_{H_{\infty}^{2}(\Omega)} & \leqslant \sigma_{19}(\xi(t)+\eta)^{1 / 2}
\end{aligned}
$$

где константа $\sigma_{17}^{p}$ зависит лишь от $р$ и $\Omega$, константы $\sigma_{18}$ и $\sigma_{19}$ зависят лишь от $\Omega$, константа $\eta \geqslant 1$ зависит лишь от данных задачи (1.1)-(1.9), указанных в формулировке теоремы 1.1. 
ДоКАЗАТЕЛЬСтво. Разумеется, (4.2) и (4.3) непосредственно следуют из (4.1) и из соответствуюших теорем вложения. В свою очередь (4.1) мы получим, используя уравнение (1.1). Имеем

$$
\begin{aligned}
\mathcal{D} \| & \nabla^{4} w(x, t)\left\|_{L_{p}(\Omega)} \leqslant\right\| w_{t t}(x, t)\left\|_{L_{p}(\Omega)}+\right\| Z(x, t) \|_{L_{p}(\Omega)} \\
& +\left\|\frac{\partial}{\partial x_{1}}\left(N_{1}(x, t) w_{x_{1}}(x, t)\right)\right\|_{L_{p}(\Omega)}+\left\|\frac{\partial}{\partial x_{2}}\left(N_{12}(x, t) w_{x_{1}}(x, t)\right)\right\|_{L_{p}(\Omega)} \\
& +\left\|\frac{\partial}{\partial x_{2}}\left(N_{2}(x, t) w_{x_{2}}(x, t)\right)\right\|_{L_{p}(\Omega)}+\left\|\frac{\partial}{\partial x_{1}}\left(N_{12}(x, t) w_{x_{2}}(x, t)\right)\right\|_{L_{p}(\Omega)} \\
& +\left\|k_{1}(x)\right\|_{L_{\infty}(\Omega)}\left\|N_{1}(x, t)\right\|_{L_{p}(\Omega)}+\left\|k_{2}(x)\right\|_{L_{\infty}(\Omega)}\left\|N_{2}(x, t)\right\|_{L_{p}(\Omega)} .
\end{aligned}
$$

Теперь правую часть полученного неравенства оценим с использованием неравенства Гёльдера и с учетом (1.11)-(1.16). Тем самым мы получим оценку

$$
\left\|\nabla^{4} w(x, t)\right\|_{L_{p}(\Omega)} \leqslant C_{1}(\sqrt{\xi(t)}+\sqrt{\eta}),
$$

где константа $C_{1}$ зависит лишь от $\Omega$ и $p$. Далее, учитьвая краевые условия (1.6) для $w$, имеем

$$
\left\|w_{x_{i} x_{j} x_{r} x_{s}}(x, t)\right\|_{L_{p}(\Omega)} \leqslant C_{2}\left\|\nabla^{4} w(x, t)\right\|_{L_{p}(\Omega)},
$$

где константа $C_{2}$ зависит лишь от $\Omega$ и $p$. Тем самым предложение 4.1 доказано.

ПРЕДЛОЖЕНИЕ 4.2. Для всех $t \in\left[0, t_{f}\right], i, j, r, s=1,2$, имеют место оценки

$$
\left\|w_{x_{i} x_{j} t}(x, t) w_{x_{r}}(x, t) w_{x_{s}}(x, t)\right\|_{L_{2}(\Omega)} \leqslant \mu_{1}^{1} \ln N(\xi(t))^{1 / 2}+\mu_{2}^{1} N^{-1 / 4}(\xi(t)+\eta)^{3 / 2}
$$

для всех $N \in \mathbb{Z}, N \geqslant 2$, где $\mu_{1}^{1}, \mu_{2}^{1}$ - константы, зависящие лишь от данных задачи (1.1)-(1.9), указанных в формулировке теоремы 1.1.

ДокаЗАТЕЛЬСТво. Имеем

$$
\begin{aligned}
\| w_{x_{i} x_{j}} t & (x, t) w_{x_{r}}(x, t) w_{x_{s}}(x, t) \|_{L_{2}(\Omega)} \\
\leqslant & \left\|w_{x_{i} x_{j} t}(x, t)\left(T_{N} Q w_{x_{r}}\right)(x, t)\left(T_{N} Q w_{x_{s}}\right)(x, t)\right\|_{L_{2}(\Omega)} \\
& +\left\|w_{x_{i} x_{j} t}(x, t)\left(T_{N} Q w_{x_{r}}\right)(x, t)\left(R_{N} Q w_{x_{s}}\right)(x, t)\right\|_{L_{2}(\Omega)} \\
& +\left\|w_{x_{i} x_{j} t}(x, t)\left(R_{N} Q w_{x_{r}}\right)(x, t)\left(T_{N} Q w_{x_{s}}\right)(x, t)\right\|_{L_{2}(\Omega)} \\
& +\left\|w_{x_{i} x_{j} t}(x, t)\left(R_{N} Q w_{x_{r}}\right)(x, t)\left(R_{N} Q w_{x_{s}}\right)(x, t)\right\|_{L_{2}(\Omega)} .
\end{aligned}
$$

Используя (3.5) и учитьвая (1.11), оценим первое слагаемое (4.6). Имеем

$$
\begin{aligned}
& \left\|w_{x_{i} x_{j} t}(x, t)\left(T_{N} Q w_{x_{r}}\right)(x, t)\left(T_{N} Q w_{x_{s}}\right)(x, t)\right\|_{L_{2}(\Omega)} \\
& \leqslant \max _{x \in D}\left|\left(T_{N} Q w_{x_{r}}\right)(x, t)\right| \max _{x \in D}\left|\left(T_{N} Q w_{x_{s}}\right)(x, t)\right|\left\|w_{x_{i} x_{j} t}(x, t)\right\|_{L_{2}(\Omega)} \\
& \leqslant C_{1} \ln N\left\|\left(Q w_{x_{r}}\right)(x, t)\right\|_{H_{2}^{1}}\left\|\left(Q w_{x_{s}}\right)(x, t)\right\|_{H_{2}^{1}}\left\|w_{x_{i} x_{j} t}(x, t)\right\|_{L_{2}(\Omega)} \\
& \leqslant C_{2} \ln N\|w(x, t)\|_{\mathrm{H}_{2}^{2}(\Omega)}^{2}(\xi(t))^{1 / 2} \leqslant C_{3} \ln N(\xi(t))^{1 / 2} .
\end{aligned}
$$


Здесь константа $C_{3}$ зависит лишш от констант $B_{2}$ и $B_{12}$ и от области $\Omega$.

Теперь, используя (3.5) и (3.6) и учитывая (1.11) и (4.2), оценим второе и третье слагаемые (4.6). Имеем

$$
\begin{aligned}
& \left\|w_{x_{i} x_{j} t}(x, t)\left(T_{N} Q w_{x_{r}}\right)(x, t)\left(R_{N} Q w_{x_{s}}\right)(x, t)\right\|_{L_{2}(\Omega)} \\
& \quad \leqslant \max _{x \in D}\left|\left(T_{N} Q w_{x_{r}}\right)(x, t)\right| \max _{x \in D}\left|\left(R_{N} Q w_{x_{s}}\right)(x, t)\right|\left\|w_{x_{i} x_{j} t}(x, t)\right\|_{L_{2}(\Omega)} \\
& \quad \leqslant C_{4} N^{-1 / 2}(\ln N)^{1 / 2}\left\|\left(Q w_{x_{r}}\right)(x, t)\right\|_{H_{2}^{1}}\left\|\left(Q w_{x_{s}}\right)(x, t)\right\|_{H_{2}^{3}}\left\|w_{x_{i} x_{j} t}(x, t)\right\|_{L_{2}(\Omega)} \\
& \quad \leqslant C_{5} N^{-1 / 2}(\ln N)^{1 / 2}\|w(x, t)\|_{\stackrel{\circ}{2}_{2}^{2}(\Omega)}\|w(x, t)\|_{H_{2}^{3}(\Omega)}\left\|w_{x_{i} x_{j} t}(x, t)\right\|_{L_{2}(\Omega)} \\
& \quad \leqslant C_{6} N^{-1 / 2}(\ln N)^{1 / 2} \xi(t)
\end{aligned}
$$

Аналогично получаем

$$
\left\|w_{x_{i} x_{j} t}(x, t)\left(R_{N} Q w_{x_{r}}\right)(x, t)\left(T_{N} Q w_{x_{s}}\right)(x, t)\right\|_{L_{2}(\Omega)} \leqslant C_{6} N^{-1 / 2}(\ln N)^{1 / 2} \xi(t) .
$$

Здесь константа $C_{6}$ зависит лиш от констант $B_{12}, B_{13}, B_{2}$ и от области $\Omega$.

Сходным образом оценим последнее слагаемое (4.6). Имеем

$$
\begin{aligned}
& \left\|w_{x_{i} x_{j} t}(x, t)\left(R_{N} Q w_{x_{r}}\right)(x, t)\left(R_{N} Q w_{x_{s}}\right)(x, t)\right\|_{L_{2}(\Omega)} \\
& \quad \leqslant \max _{x \in D}\left|\left(R_{N} Q w_{x_{r}}\right)(x, t)\right| \max _{x \in D}\left|\left(R_{N} Q w_{x_{s}}\right)(x, t)\right|\left\|w_{x_{i} x_{j} t}(x, t)\right\|_{L_{2}(\Omega)} \\
& \quad \leqslant C_{7} N^{-1}\|w(x, t)\|_{H_{2}^{3}(\Omega)}^{2}\left\|w_{x_{i} x_{j} t}(x, t)\right\|_{L_{2}(\Omega)} \leqslant C_{8} N^{-1}(\xi(t))^{3 / 2}
\end{aligned}
$$

Константа $C_{8}$ зависит лишь от константы $B_{9}$ и от области $\Omega$. Оценки (4.6)-(4.10) дают (4.5). Предложение доказано.

Теперь мы можем оценить $A_{8}(t)$.

ПРЕДЛОЖЕНИЕ 4.3. Для всех $t \in\left[0, t_{f}\right]$ u всех $N \in \mathbb{Z}, N \geqslant 2$, имеет место следующая оценка:

$$
A_{8}(t) \leqslant \mu_{1}^{1} \ln N \xi(t)+\mu_{2}^{1} N^{-1 / 4}(\xi(t)+\eta)^{2} .
$$

ДоКАЗАТЕЛЬСТВо легко следует из оценки (4.5). Действительно, применив неравенство Шварца, получаем

$$
A_{8}(t) \leqslant\left\|w_{x_{i} x_{j} t}(x, t) w_{x_{r}}(x, t) w_{x_{s} t}(x, t)\right\|_{L_{2}(\Omega)}\left\|w_{t t}(x, t)\right\|_{L_{2}(\Omega)} .
$$

Из (4.12) и (4.5) следует (4.11). Предложение доказано. 
ПРЕДЛОЖЕНИЕ 4.4. Для всех $t \in\left[0, t_{f}\right], i, j, r, s=1,2$ имеют место оченки

$$
\begin{aligned}
& \left\|w_{x_{i} x_{j}}(x, t) w_{x_{r}}(x, t) w_{x_{s} t}(x, t)\right\|_{L_{2}(\Omega)} \\
& \quad \leqslant \mu_{1}^{2} \ln N(\xi(t))^{1 / 2}+\mu_{2}^{2} N^{-1 / 2}(\xi(t)+\eta)^{3 / 2}
\end{aligned}
$$

для всех $N \in \mathbb{Z}, \quad N \geqslant 2$, где $\mu_{1}^{2}, \mu_{2}^{2}$ - константы, зависящие лишь от данных задачи (1.1)-(1.9), указанных в формулировке теоремы 1.1, и от области $\Omega$.

ДокАЗАТЕЛЬСтво. Аналогично (4.6) имеем

$$
\begin{aligned}
&\left\|w_{x_{i} x_{j}}(x, t) w_{x_{r}}(x, t) w_{x_{s} t}(x, t)\right\|_{L_{2}(\Omega)} \\
& \leqslant\left\|w_{x_{i} x_{j}}(x, t)\left(T_{N} Q w_{x_{r}}\right)(x, t)\left(T_{N} Q w_{x_{s} t}\right)(x, t)\right\|_{L_{2}(\Omega)} \\
&+\left\|w_{x_{i} x_{j}}(x, t)\left(T_{N} Q w_{x_{r}}\right)(x, t)\left(R_{N} Q w_{x_{s} t}\right)(x, t)\right\|_{L_{2}(\Omega)} \\
&+\left\|w_{x_{i} x_{j}}(x, t)\left(R_{N} Q w_{x_{r}}\right)(x, t)\left(T_{N} Q w_{x_{s} t}\right)(x, t)\right\|_{L_{2}(\Omega)} \\
&+\left\|w_{x_{i} x_{j}}(x, t)\left(R_{N} Q w_{x_{r}}\right)(x, t)\left(R_{N} Q w_{x_{s} t}\right)(x, t)\right\|_{L_{2}(\Omega)} .
\end{aligned}
$$

Действуя так же, как и при получении оценки (4.7), получаем

$$
\begin{aligned}
& \left\|w_{x_{i} x_{j}}(x, t)\left(T_{N} Q w_{x_{r}}\right)(x, t)\left(T_{N} Q w_{x_{s} t}\right)(x, t)\right\|_{L_{2}(\Omega)} \\
& \quad \leqslant \max _{x \in D}\left|\left(T_{N} Q w_{x_{r}}\right)(x, t)\right| \max _{x \in D}\left|\left(T_{N} Q w_{x_{s} t}\right)(x, t)\right|\left\|w_{x_{i} x_{j}}(x, t)\right\|_{L_{2}(\Omega)} \\
& \leqslant C_{1} \ln N\|w(x, t)\|_{\mathrm{O}_{2}^{2}(\Omega)}^{2}\left\|w_{t}(x, t)\right\|_{\stackrel{O}{2}_{2}^{2}(\Omega)} \leqslant C_{2} \ln N(\xi(t))^{1 / 2} .
\end{aligned}
$$

Здесь константа $C_{2}$ зависит лишь от констант $B_{12}$ и $B_{13}$ и от области $\Omega$.

Теперь, используя (3.5), (4.3), (3.4), с учетом (1.11) получаем

$$
\begin{aligned}
& \left\|w_{x_{i} x_{j}}(x, t)\left(T_{N} Q w_{x_{r}}\right)(x, t)\left(R_{N} Q w_{x_{s}}\right)(x, t)\right\|_{L_{2}(\Omega)} \\
& \quad \leqslant \max _{x \in D}\left|\left(T_{N} Q w_{x_{r}}\right)(x, t)\right|\left\|w_{x_{i} x_{j}}(x, t)\right\|_{L_{\infty}(\Omega)}\left\|\left(R_{N} Q w_{x_{s}}\right)(x, t)\right\|_{L_{2}(\Omega)} \\
& \quad \leqslant C_{3}(\ln N)^{1 / 2} N^{-1}(\xi(t)+\eta) .
\end{aligned}
$$

Здесь константа $C_{3}$ зависит лишь от констант $B_{12}, B_{13}$ и $B_{2}$ и от области $\Omega$.

Используя (4.3), (3.5), (3.4), имеем

$$
\begin{aligned}
& \left\|w_{x_{i} x_{j}}(x, t)\left(R_{N} Q w_{x_{r}}\right)(x, t)\left(T_{N} Q w_{x_{s} t}\right)(x, t)\right\|_{L_{2}(\Omega)} \\
& \quad \leqslant \max _{x \in D}\left|\left(T_{N} Q w_{x_{s} t}\right)(x, t)\right|\left\|w_{x_{i} x_{j}}(x, t)\right\|_{L_{\infty}(\Omega)}\left\|\left(R_{N} Q w_{x_{r}}\right)(x, t)\right\|_{L_{2}(\Omega)} \\
& \quad \leqslant C_{4}(\ln N)^{1 / 2} N^{-1}(\xi(t)+\eta) .
\end{aligned}
$$


Здесь константа $C_{4}$ зависит лишь от констант $B_{12}$ и $\sigma_{19}$ и от области $\Omega$.

Теперь, используя (4.3), (3.6), (3.4), получаем

$$
\begin{aligned}
& \left\|w_{x_{i} x_{j}}(x, t)\left(R_{N} Q w_{x_{r}}\right)(x, t)\left(R_{N} Q w_{x_{s}} t\right)(x, t)\right\|_{L_{2}(\Omega)} \\
& \quad \leqslant \max _{x \in D}\left|\left(R_{N} Q w_{x_{r}}\right)(x, t)\right|\left\|w_{x_{i} x_{j}}(x, t)\right\|_{L_{\infty}(\Omega)}\left\|\left(R_{N} Q w_{x_{s}}\right)(x, t)\right\|_{L_{2}(\Omega)} \\
& \quad \leqslant C_{5} N^{-3 / 2}(\xi(t)+\eta)^{3 / 2} .
\end{aligned}
$$

Здесь константа $C_{5}$ зависит лишь от констант $B_{13}$ и $B_{2}$ и от области $\Omega$. Оценки (4.15)-(4.18) дают (4.13). Предложение доказано.

Оценим $A_{9}(t)$.

ПРЕДЛОЖЕНИЕ 4.5. Для всех $N \in \mathbb{Z}, \quad N \geqslant 2$, имеет место следующая оченка:

$$
A_{9}(t) \leqslant \mu_{1}^{2} \ln N \xi(t)+\mu_{2}^{2} N^{-1 / 2}(\xi(t)+\eta)^{2} .
$$

Доказательство полностью аналогично доказательству предложения 4.3.

\section{§5. Оценка $A_{10}(t)$}

Рассмотрим однородную краевую задачу для эллиптической системы уравнений

$$
\begin{gathered}
\Delta u+\frac{1+\mu}{1-\mu} \theta_{x_{1}}=f_{1}, \\
\Delta v+\frac{1+\mu}{1-\mu} \theta_{x_{2}}=f_{2}, \\
\left.u\right|_{\Gamma}=\left.v\right|_{\Gamma}=0
\end{gathered}
$$

ЗАмечАниЕ 5.1. Обобщенное решение $u, v$ задачи (5.1)-(5.3), удовлетворяющее интегральному соотношению

$$
\int_{\Omega}\left[\left(-\Delta u-\frac{1+\mu}{1-\mu} \theta_{x_{1}}\right) u_{0}+\left(-\Delta v-\frac{1+\mu}{1-\mu} \theta_{x_{2}}\right) v_{0}+f_{1} u_{0}+f_{2} v_{0}\right] d x=0
$$

для любых $u_{0}, v_{0} \in \stackrel{\circ}{H} \frac{1}{2}(\Omega)$, существует и имеет следуюшую оценку:

$$
\sum_{i=1}^{2}\left(\left\|u_{x_{i}}\right\|_{L_{q}(\Omega)}+\left\|v_{x_{i}}\right\|_{L_{q}(\Omega)}\right) \leqslant C\left(\left\|f_{1}\right\|_{L_{p}(\Omega)}+\left\|f_{2}\right\|_{L_{p}(\Omega)}\right), \quad q<\frac{2 p}{2-p}
$$

где константа $C$ не зависит от $u, v$ (см. [1, с. 765,766$])$.

Обозначив через $F_{1}, F_{2}$ соответственно правые части $(1.4),(1.5)$, отметим, что $u_{t}, v_{t}$ являются решением (5.1)-(5.3) при $f_{1}=F_{1 t}, f_{2}=F_{2 t}$.

Теперь оценим $F_{1 t}, F_{2 t}$. 
ПРЕДЛОЖЕНИЕ 5.2. Для всех $t \in\left[0, t_{f}\right]$ имеет место следующая оценка:

$$
\left\|F_{1}(x, t)\right\|_{L_{\frac{3}{2}}(\Omega)}+\left\|F_{2}(x, t)\right\|_{L_{\frac{3}{2}}(\Omega)} \leqslant \sigma_{20}(\xi(t)+\eta)^{1 / 2},
$$

где константа $\sigma_{20}$ зависит лишь от данных задачи (1.1)-(1.9), указанных в формулировке теоремь 1.1, и от области $\Omega$.

ДокАЗАТЕЛЬСТво. Учитывая вид правых частей (1.4) и (1.5), легко получим

$$
\begin{gathered}
\left\|F_{1 t}(x, t)\right\|_{L_{\frac{3}{2}}(\Omega)}+\left\|F_{2 t}(x, t)\right\|_{L_{\frac{3}{2}}(\Omega)} \leqslant C_{1}(\xi(t)+\eta)^{1 / 2} \\
+C_{2}\left[\sum _ { i , j , r = 1 } ^ { 2 } \left(\left\|w_{x_{i} x_{j} t}(x, t) w_{x_{r}}(x, t)\right\|_{L_{\frac{3}{2}}(\Omega)}\right.\right. \\
\left.\left.+\| w_{x_{i} x_{j}}(x, t) w_{x_{r} t}(x, t)\right) \|_{L_{\frac{3}{2}}(\Omega)}\right]
\end{gathered}
$$

где константы $C_{1}$ и $C_{2}$ зависят лишь от коэффициентов $\mu, k_{1}, k_{2}$ из (1.4), (1.5). Используя неравенство Гёльдера и (1.14), получаем

$$
\begin{aligned}
& \left\|w_{x_{i} x_{j} t}(x, t) w_{x_{r}}(x, t)\right\|_{L_{\frac{3}{2}}(\Omega)} \\
& \quad \leqslant\left\|w_{x_{i} x_{j} t}(x, t)\right\|_{L_{2}(\Omega)}\left\|w_{x_{r}}(x, t)\right\|_{L_{6}(\Omega)} \leqslant C_{3}(\xi(t))^{1 / 2} .
\end{aligned}
$$

Далее, используя неравенство Гёльдера, ограниченное вложение $H_{2}^{1}(\Omega)$ в $L_{6}(\Omega)$ и (1.11), имеем

$$
\begin{aligned}
& \left\|w_{x_{i} x_{j}}(x, t) w_{x_{r} t}(x, t)\right\|_{L_{\frac{3}{2}}(\Omega)} \\
& \leqslant\left\|w_{x_{i} x_{j}}(x, t)\right\|_{L_{2}(\Omega)}\left\|w_{x_{r} t}(x, t)\right\|_{L_{6}(\Omega)} \leqslant C_{4}(\xi(t))^{1 / 2} .
\end{aligned}
$$

Здесь $C_{3}$ и $C_{4}$ зависят только от констант $B_{5}^{6}$ и $B_{2}$. Из (5.7)-(5.9) следует (5.6). Предложение доказано.

ПрЕДЛОЖЕНИЕ 5.3. Для всех $t \in\left[0, t_{f}\right]$ имеет место следующая оченка:

$$
\sum_{i=1}^{2}\left(\left\|u_{x_{i}}(x, t)\right\|_{L_{2}(\Omega)}+\left\|v_{x_{i} t}(x, t)\right\|_{L_{2}(\Omega)}\right) \leqslant \sigma_{21}(\xi(t)+\eta)^{1 / 2}
$$

где константа $\sigma_{21}$ зависит лищь от данных задачи (1.1)-(1.9), указанных в формулировке теоремы 1.1, и от области $\Omega$.

Доказательство непосредственно следует из (5.5) и (5.6).

Теперь мы уже можем оценить $A_{10}(t)$.

ПрЕДЛОЖЕНИЕ 5.4. Для всех $t \in\left[0, t_{f}\right]$ имеет место следующая оценка:

$$
A_{10}(t) \leqslant \sigma_{22}(\xi(t)+\eta)
$$

где константа $\sigma_{22}$ зависит лишь от данных задачи (1.1)-(1.9), указанных в формулировке теоремы 1.1, и от области $\Omega$.

Доказательство (после применения к каждому слагаемому (2.13) неравенства Шварца) следует теперь из оценки (5.10). 
§6. Оценки $A_{11}(t)$ и $A_{12}(t)$

Сначала придется указать более продвинутые оценки решений краевой задачи (5.1)-(5.3).

ПРЕДЛОЖЕНИЕ 6.1. Пусть $f_{1}, f_{2} \in H_{p}^{r}(\Omega) \cap L_{2}(\Omega), \quad r=0,1,2, p>1$.

Тогда существует единственное решение краевой задачи (5.1)-(5.3) такое, что $и, v \in H_{p}^{r+2}(\Omega) \cap \stackrel{\circ}{H} \frac{1}{2}(\Omega)$, причем имеет место оценка:

$$
\|u\|_{H_{p}^{r+2}(\Omega)}+\|v\|_{H_{p}^{r+2}(\Omega)} \leqslant C\left(\left\|f_{1}\right\|_{H_{p}^{r}(\Omega)}+\left\|f_{2}\right\|_{H_{p}^{r}(\Omega)}\right),
$$

где константа $C$ зависит лишь от $r, p, \Omega$.

ДокАЗАТЕЛЬСТво. Для каждого $\varepsilon \in[0,1]$ рассмотрим краевую задачу:

$$
\begin{gathered}
\Delta u^{\varepsilon}+\varepsilon \frac{1+\mu}{1-\mu} \theta_{x_{1}}^{\varepsilon}=f_{1}, \\
\Delta v^{\varepsilon}+\varepsilon \frac{1+\mu}{1-\mu} \theta_{x_{2}}^{\varepsilon}=f_{2}, \\
\left.u^{\varepsilon}\right|_{\Gamma}=\left.v^{\varepsilon}\right|_{\Gamma}=0 .
\end{gathered}
$$

Дифференциальные операторы из левых частей (6.2) и (6.3) составляют коэрцитивную систему, поскольку удовлетворяют очевидным образом условиям теоремы 11.3 из [5, с. 159]. Более того, константа в неравенстве коэрцитивности может быть выбрана не зависящей от $\varepsilon$, как это следует, например, из доказательства теоремы 11.3. Теперь мы можем завершить доказательство предложения 6.1, применив метод продолжения по параметру $\varepsilon$ так, как это, например, было сделано в [7, с. 149-150]. Предложение доказано.

Перед тем как оценить $A_{11}(t)$, оценим вторые производные $u$ и $v$.

ПРЕДЛОЖЕНИЕ 6.2. Для всех $t \in\left[0, t_{f}\right]$ имеет место оценка

$$
\begin{aligned}
& \sum_{i, j=1}^{2}\left(\left\|u_{x_{i} x_{j}}(x, t)\right\|_{L_{2}(\Omega)}+\left\|v_{x_{i} x_{j}}(x, t)\right\|_{L_{2}(\Omega)}\right) \\
& \leqslant \mu_{1}^{3}(\ln N)^{1 / 2}+\mu_{2}^{3} N^{-1 / 2}(\xi(t))^{1 / 2}
\end{aligned}
$$

при $N \in \mathbb{Z}, \quad N \geqslant 2$, где $\mu_{1}^{3}, \mu_{2}^{3}$ - константы, зависящие лишь от данных задачи (1.1)-(1.9), указанных в формулировке теоремы 1.1, и от области $\Omega$. 
ДоКАЗАТЕЛЬСТВо. Воспользуемся оценкой (6.1) с учетом вида правых частей (1.4) и (1.6) и априорной оценки (1.14). Имеем

$$
\begin{aligned}
& \sum_{i, j=1}^{2}\left(\left\|u_{x_{i} x_{j}}(x, t)\right\|_{L_{2}(\Omega)}+\left\|v_{x_{i} x_{j}}(x, t)\right\|_{L_{2}(\Omega)}\right) \\
& \quad \leqslant C_{1}+C_{2} \sum_{i, j, r=1}^{2}\left\|w_{x_{i} x_{j}}(x, t) w_{x_{r}}(x, t)\right\|_{L_{2}(\Omega)},
\end{aligned}
$$

где константы $C_{1}$ и $C_{2}$ зависят лишь от коэффициентов $(1.4),(1.5)$, величины норм $X$ и $Y$ в $L_{2}\left(\Omega \times\left[0, t_{f}\right]\right)$, константы $B_{5}^{2}$.

Далее, аналогично (4.6) имеем

$$
\begin{aligned}
\left\|w_{x_{i} x_{j}}(x, t) w_{x_{r}}(x, t)\right\|_{L_{2}(\Omega)} \leqslant & \left\|w_{x_{i} x_{j}}(x, t)\left(T_{N} Q w_{x_{r}}\right)(x, t)\right\|_{L_{2}(\Omega)} \\
& +\left\|w_{x_{i} x_{j}}(x, t)\left(R_{N} Q w_{x_{r}}\right)(x, t)\right\|_{L_{2}(\Omega)} .
\end{aligned}
$$

Теперь аналогично (4.7) получаем

$$
\left\|w_{x_{i} x_{j}}(x, t)\left(T_{N} Q w_{x_{r}}\right)(x, t)\right\|_{L_{2}(\Omega)} \leqslant C_{3}(\ln N)^{1 / 2} .
$$

Аналогично (4.11) имеем

$$
\left\|w_{x_{i} x_{j}}(x, t)\left(R_{N} Q w_{x_{r}}\right)(x, t)\right\|_{L_{2}(\Omega)} \leqslant C_{4} N^{-1 / 2}(\xi(t))^{1 / 2}
$$

Константы $C_{3}$ и $C_{4}$ зависят лиш от констант $B_{12}, B_{13}, B_{2}$ и от области $\Omega$. Оценки (6.6)-(6.9) дают (6.5). Предложение доказано.

Теперь оценим $A_{11}(t)$.

ПРЕДЛОЖЕНИЕ 6.3. Для всех $t \in\left[0, t_{f}\right]$ u всех $N \in \mathbb{Z}, N \geqslant 2$, имеет место оценка

$$
A_{11}(t) \leqslant \mu_{1}^{4} \ln N \xi(t)+\mu_{2}^{4} N^{-1 / 4}(\xi(t)+\eta)^{2},
$$

где $\mu_{1}^{4}, \mu_{2}^{4}$ - константы, зависящие лищь от данных задачи (1.1)-(1.9), указанных в формулировке теоремы 1.1, и от области $\Omega$.

ДокАзАТЕЛЬСтво. Так же, как и ранее, имеем

$$
\begin{aligned}
& \left\|w_{x_{i} t}(x, t) u_{x_{j} x_{r}}(x, t) w_{t t}(x, t)\right\|_{L_{1}(\Omega)} \\
& \leqslant\left\|\left(T_{N} Q w_{x_{i} t}\right)(x, t) u_{x_{j} x_{r}}(x, t) w_{t t}(x, t)\right\|_{L_{1}(\Omega)} \\
& \quad+\left\|\left(R_{N} Q w_{x_{i} t}\right)(x, t) u_{x_{j} x_{r}}(x, t) w_{t t}(x, t)\right\|_{L_{1}(\Omega)} .
\end{aligned}
$$


Теперь, используя (3.5), неравенство Шварца, оценку (6.5), получаем

$$
\begin{aligned}
& \left\|\left(T_{N} Q w_{x_{i} t}\right)(x, t) u_{x_{j} x_{r}}(x, t) w_{t t}(x, t)\right\|_{L_{1}(\Omega)} \\
& \quad \leqslant \max _{x \in D}\left|\left(T_{N} Q w_{x_{i} t}\right)(x, t)\right|\left\|u_{x_{j} x_{r}}(x, t)\right\|_{L_{2}(\Omega)}\left\|w_{t t}(x, t)\right\|_{L_{2}(\Omega)} \\
& \quad \leqslant C_{1} \ln N \xi(t)+C_{2}(\ln N)^{1 / 2} N^{-1 / 2}(\xi(t))^{3 / 2} .
\end{aligned}
$$

Константы $C_{1}$ и $C_{2}$ зависят лишь от констант $B_{12}, \mu_{1}^{3}, \mu_{2}^{3}$ и от области $\Omega$. Из теоремы вложения, оценки (6.1), вида правых частей (1.4), (1.5), неравенства Гёльдера, опять из теорем вложения, оценок (4.1) и (4.3) имеем

$$
\begin{aligned}
& \left\|u_{x_{j} x_{r}}(x, t)\right\|_{L_{\infty}(\Omega)}+\left\|v_{x_{j} x_{r}}(x, t)\right\|_{L_{\infty}(\Omega)} \\
& \leqslant C_{3}\left(\|u(x, t)\|_{H_{2}^{4}(\Omega)}+\|v(x, t)\|_{H_{2}^{4}(\Omega)}\right) \\
& \leqslant C_{4}(\xi(t)+\eta)^{1 / 2}+C_{5} \sum_{i, j, k, r, s=1}^{2}\left(\left\|w_{x_{i} x_{j} x_{k} x_{r}}(x, t) w_{x_{s}}(x, t)\right\|_{L_{2}(\Omega)}\right. \\
& \left.\quad+\left\|w_{x_{i} x_{j} x_{k}}(x, t) w_{x_{r} x_{s}}(x, t)\right\|_{L_{2}(\Omega)}\right) \\
& \leqslant C_{4}(\xi(t)+\eta)^{1 / 2}+C_{5} \sum_{i, j, k, r, s=1}^{2}\left(\left\|w_{x_{s}}(x, t)\right\|_{L_{\infty}(\Omega)}\left\|w_{x_{i} x_{j} x_{k} x_{r}}(x, t)\right\|_{L_{2}(\Omega)}\right. \\
& \left.\quad+\left\|w_{x_{r} x_{s}}(x, t)\right\|_{L_{\infty}(\Omega)}\left\|w_{x_{i} x_{j} x_{k}}(x, t)\right\|_{L_{2}(\Omega)}\right) \\
& \leqslant C_{4}(\xi(t)+\eta)^{1 / 2}+C_{6}(\xi(t)+\eta) \leqslant C_{7}(\xi(t)+\eta) .
\end{aligned}
$$

Константа $C_{7}$ зависит лишь от коэффициентов уравнений (1.1)-(1.5) и от области $\Omega$.

Теперь, учитывая (6.13) и (3.3), с использованием неравенства Шварца получаem

$$
\begin{aligned}
& \left\|\left(R_{N} Q w_{x_{i} t}\right)(x, t) u_{x_{j} x_{r}}(x, t) w_{t t}(x, t)\right\|_{L_{1}(\Omega)} \\
& \quad \leqslant\left\|u_{x_{j} x_{r}}(x, t)\right\|_{L_{\infty}(\Omega)}\left\|\left(R_{N} Q w_{x_{i} t}\right)(x, t)\right\|_{L_{2}(\Omega)}\left\|w_{t t}(x, t)\right\|_{L_{2}(\Omega)} \\
& \quad \leqslant C_{8} N^{-1}(\xi(t)+\eta)^{2} .
\end{aligned}
$$

Константа $C_{8}$ зависит от области $\Omega$. Из (6.11)-(6.14) следует (6.10). Предложение доказано.

ПРеДЛОЖЕНИЕ 6.4. Для всех $t \in\left[0, t_{f}\right] u$ всех $N \in \mathbb{Z}, N \geqslant 2$, имеет место следующая оценка:

$$
A_{12}(t) \leqslant \mu_{1}^{5} \ln N \xi(t)+\mu_{2}^{5} N^{-1 / 3}(\xi(t)+\eta)^{3 / 2},
$$

где $\mu_{1}^{5}, \mu_{2}^{5}$ - константы, зависящие лищь от данных задачи (1.1)-(1.9), указанных в формулировке теоремы 1.1, и от области $\Omega$. 
ДоКАЗАТЕЛЬСТВО. Применим привычный прием. Имеем

$$
\begin{aligned}
& \left\|w_{x_{i} x_{j} t}(x, t) u_{x_{r}}(x, t) w_{t t}(x, t)\right\|_{L_{1}(\Omega)} \\
& \leqslant \\
& \quad\left\|w_{x_{i} x_{j} t}(x, t)\left(T_{N} Q u_{x_{r}}\right)(x, t) w_{t t}(x, t)\right\|_{L_{1}(\Omega)} \\
& \quad+\left\|w_{x_{i} x_{j} t}(x, t)\left(R_{N} Q u_{x_{r}}\right)(x, t) w_{t t}(x, t)\right\|_{L_{1}(\Omega)} .
\end{aligned}
$$

Далее, учитьвая (3.5) и (6.5), с использованием неравенства Шварца получаем

$$
\begin{aligned}
& \left\|w_{x_{i} x_{j} t}(x, t)\left(T_{N} Q u_{x_{r}}\right)(x, t) w_{t t}(x, t)\right\|_{L_{1}(\Omega)} \\
& \quad \leqslant \max _{x \in D}\left|\left(T_{N} Q u_{x_{r}}\right)\right|\left\|w_{t}(x, t)\right\|_{\stackrel{O}{2}_{2}^{2}(\Omega)}\left\|w_{t t}(x, t)\right\|_{L_{2}(\Omega)} \\
& \leqslant C_{1}(\ln N)^{1 / 2}\left\|u_{x_{r}}(x, t)\right\|_{H_{2}^{1}(\Omega)} \xi(t) \\
& \leqslant C_{2} \ln N \xi(t)+C_{3}(\ln N)^{1 / 2} N^{-1 / 2}(\xi(t))^{3 / 2} .
\end{aligned}
$$

Здесь константы $C_{2}$ и $C_{3}$ зависят лишш от констант $B_{12}, B_{3}, \mu_{1}^{3}, \mu_{2}^{3}$ и от области $\Omega$.

Теперь с учетом (3.4) и (6.13) оценим второе слагаемое правой части (6.16). Используя вложение $H_{2}^{2}(\Omega)$ в $L_{\infty}(\Omega)$, имеем

$$
\begin{aligned}
& \left\|w_{x_{i} x_{j} t}(x, t)\left(R_{N} Q u_{x_{r}}\right)(x, t) w_{t t}(x, t)\right\|_{L_{1}(\Omega)} \\
& \quad \leqslant \max _{x \in D}\left|\left(R_{N} Q u_{x_{r}}\right)\right|\left\|w_{t}(x, t)\right\|_{H_{2}^{2}(\Omega)}\left\|w_{t t}(x, t)\right\|_{L_{2}(\Omega)} \\
& \leqslant C_{4}\left\|\left(R_{N} Q u_{x_{r}}\right)(x, t)\right\|_{H_{2}^{2}(\Omega)} \xi(t) \\
& \leqslant C_{5} N^{-1}\|u(x, t)\|_{H_{2}^{4}(\Omega)} \leqslant C_{6} N^{-1}(\xi(t)+\eta) .
\end{aligned}
$$

Константа $C_{6}$ зависит лишь от коэффициентов уравнений (1.1)-(1.5) и от области $\Omega$. Из (6.16)-(6.18) следует (6.15). Предложение доказано.

\section{$\S 7$. Оценка $A_{13}(t)$ и $A_{14}(t)$}

Предварительно нам придется доказать следуюшую вспомогательную оценку.

ПРЕДЛОЖЕНИЕ 7.1. Для всех $t \in\left[0, t_{f}\right]$ имеет место следующая оценка:

$$
\begin{aligned}
& \sum_{i, j=1}^{2}\left(\left\|u_{x_{i} x_{j} t}(x, t)\right\|_{L_{2}(\Omega)}+\left\|v_{x_{i} x_{j} t}(x, t)\right\|_{L_{2}(\Omega)}\right) \\
& \quad \leqslant \mu_{1}^{6}(\ln N)^{1 / 2}(\xi(t)+\eta)^{1 / 2}+\mu_{2}^{6} N^{-1 / 2}(\xi(t)+\eta)
\end{aligned}
$$

где константы $\mu_{1}^{6}, \mu_{2}^{6}$ зависят лишь от данньх задачи (1.1)-(1.9), указанньх в формулировке теоремы 1.1, и от области $\Omega$. 
ДоКАЗАТЕЛЬСТВо. Из оценки (6.1) и вида правых частей уравнений (1.4) и (1.5) аналогично (5.7) имеем

$$
\begin{aligned}
& \sum_{i, j=1}^{2}\left(\left\|u_{x_{i} x_{j} t}(x, t)\right\|_{L_{2}(\Omega)}+\left\|v_{x_{i} x_{j} t}(x, t)\right\|_{L_{2}(\Omega)}\right) \\
& \leqslant C_{1}\left(\left\|F_{1 t}(x, t)\right\|_{L_{2}(\Omega)}+\left\|F_{2 t}(x, t)\right\|_{L_{2}(\Omega)}\right) \\
& \leqslant C_{2}(\xi(t)+\eta)^{1 / 2}+C_{3}\left(\sum_{i, j, r=1}^{2}\left\|w_{x_{i} x_{j} t}(x, t) w_{x_{r}}(x, t)\right\|_{L_{2}(\Omega)}\right. \\
& \left.+\left\|w_{x_{i} x_{j}}(x, t) w_{x_{r} t}(x, t)\right\|_{L_{2}(\Omega)}\right)
\end{aligned}
$$

где константы $C_{2}$ и $C_{3}$ зависят лишь от коэффициентов уравнений (1.4) и (1.5).

Теперь оценим оставшиеся неоцененными величины из правой части (7.2). Делается это уже привычным образом. Учитывая (3.3), (3.5), (3.6), (1.11) и $(4.2),(4.3)$, имеем

$$
\begin{aligned}
& \left\|w_{x_{i} x_{j} t}(x, t) w_{x_{r}}(x, t)\right\|_{L_{2}(\Omega)} \\
& \quad \leqslant\left\|w_{x_{i} x_{j} t}(x, t)\left(T_{N} Q w_{x_{r}}\right)(x, t)\right\|_{L_{2}(\Omega)}+\left\|w_{x_{i} x_{j} t}(x, t)\left(R_{N} Q w_{x_{r}}\right)(x, t)\right\|_{L_{2}(\Omega)} \\
& \quad \leqslant C_{4}(\ln N)^{1 / 2}(\xi(t))^{1 / 2}+C_{5} N^{-1 / 2}(\xi(t)+\eta) \\
& \left\|w_{x_{i} x_{j}}(x, t) w_{x_{r} t}(x, t)\right\|_{L_{2}(\Omega)} \\
& \quad \leqslant\left\|w_{x_{i} x_{j}}(x, t)\left(T_{N} Q w_{x_{r}}\right)(x, t)\right\|_{L_{2}(\Omega)}+\left\|w_{x_{i} x_{j}}(x, t)\left(R_{N} Q w_{x_{r} t}\right)(x, t)\right\|_{L_{2}(\Omega)} \\
& \quad \leqslant C_{6}(\ln N)^{1 / 2}(\xi(t))^{1 / 2}+C_{7}\left\|w_{x_{i} x_{j}}(x, t)\right\|_{L_{\infty}(\Omega)}\left\|\left(R_{N} Q w_{x_{r} t}\right)(x, t)\right\|_{L_{2}(\Omega)} \\
& \quad \leqslant C_{6}(\ln N)^{1 / 2}(\xi(t))^{1 / 2}+C_{8} N^{-1}(\xi(t)+\eta)
\end{aligned}
$$

Константы $C_{4}, C_{5}, C_{6}, C_{8}$ зависят лишь от констант $B_{12}, B_{13}, B_{2}$ и от области $\Omega$. Из (7.2)-(7.4) следует (7.1). Предложение доказано.

Оценим $A_{13}(t)$.

ПреДЛОЖЕнИЕ 7.2. Для всех $t \in\left[0, t_{f}\right]$ u всех $N \in \mathbb{Z}, N \geqslant 2$, имеет место следующая оченка:

$$
A_{13}(t) \leqslant \mu_{1}^{7} \ln N(\xi(t)+\eta)+\mu_{2}^{7} N^{-1 / 4}(\xi(t)+\eta)^{2},
$$

где $\mu_{1}^{7}, \mu_{2}^{7}$ - константьи, зависящие лищь от данньх задачи (1.1)-(1.9), указанных в формулировке теоремы 1.1, и от области $\Omega$. 
ДокАЗАТЕЛЬСтво. Учитывая (3.3), (3.6), (1.11), (7.1), (4.2), получаем

$$
\begin{aligned}
&\left\|w_{x_{i}}(x, t) u_{x_{j} x_{r} t}(x, t) w_{t t}(x, t)\right\|_{L_{1}(\Omega)} \\
& \leqslant\left\|\left(T_{N} Q w_{x_{i}}\right)(x, t) u_{x_{j} x_{r} t}(x, t) w_{t t}(x, t)\right\|_{L_{1}(\Omega)} \\
&+\left\|\left(R_{N} Q w_{x_{i}}\right)(x, t) u_{x_{j} x_{r} t}(x, t) w_{t t}(x, t)\right\|_{L_{1}(\Omega)} \\
& \leqslant\left(\max _{x \in D}\left|\left(T_{N} Q w_{x_{i}}\right)(x, t)\right|\right. \\
& \quad\left.+\max _{x \in D}\left|\left(R_{N} Q w_{x_{i}}\right)(x, t)\right|\right)\left\|w_{x_{j} x_{r} t}(x, t)\right\|_{L_{2}(\Omega)}\left\|w_{t t}(x, t)\right\|_{L_{2}(\Omega)} \\
& \leqslant C_{1}(\ln N)^{1 / 2}+C_{2} N^{-1 / 2}(\xi(t)+\eta)^{1 / 2}\left(\mu_{1}^{6}(\ln N)^{1 / 2}(\xi(t)+\eta)^{1 / 2}\right. \\
&\left.+\mu_{2}^{6} N^{-1 / 2}(\xi(t)+\eta)\right)(\xi(t))^{1 / 2} .
\end{aligned}
$$

$\mathrm{B}(7.6)$ константы $C_{1}$ и $C_{2}$ зависят лиш от констант $B_{12}, B_{13}$ и от области $\Omega$. Оценка (7.5) непосредственно следует из (7.6). Предложение доказано.

Наконец, оценим $A_{14}(t)$.

ПРЕДЛОЖЕНИЕ 7.3. Для всех $t \in\left[0, t_{f}\right]$ и всех $N \in \mathbb{Z}, N \geqslant 2$, имеет место следующая оченка:

$$
A_{14}(t) \leqslant \mu_{1}^{8} \ln N(\xi(t)+\eta)+\mu_{2}^{8} N^{-1 / 4}(\xi(t)+\eta)^{2},
$$

где $\mu_{1}^{8}, \mu_{2}^{8}$ - константь, зависящие лищь от данньх задачи (1.1)-(1.9), указанных в формулировке теоремы 1.1, и от области $\Omega$.

ДоКАЗАТЕЛЬСТВО. Имеем

$$
\begin{aligned}
& \left\|w_{x_{i} x_{j}}(x, t) u_{x_{r} t}(x, t) w_{t t}(x, t)\right\|_{L_{1}(\Omega)} \\
& \leqslant\left\|w_{x_{i} x_{j}}(x, t)\left(T_{N} Q u_{x_{r} t}\right)(x, t) w_{t t}(x, t)\right\|_{L_{1}(\Omega)} \\
& \quad+\left\|w_{x_{i} x_{j}}(x, t)\left(R_{N} Q u_{x_{r} t}\right)(x, t) w_{t t}(x, t)\right\|_{L_{1}(\Omega)} .
\end{aligned}
$$

Далее,

$$
\begin{aligned}
& \left\|w_{x_{i} x_{j}}(x, t)\left(T_{N} Q u_{x_{r} t}\right)(x, t) w_{t t}(x, t)\right\|_{L_{1}(\Omega)} \\
& \quad \leqslant \max _{x \in D}\left|\left(T_{N} Q u_{x_{r} t}\right)(x, t)\right|\left\|w_{x_{i} x_{j}}(x, t)\right\|_{L_{\infty}(\Omega)}\left\|w_{t t}(x, t)\right\|_{L_{2}(\Omega)} \\
& \quad \leqslant C_{1}(\ln N)^{1 / 2}\left\|u_{x_{r} t}(x, t)\right\|_{H_{2}^{1}(\Omega)}(\xi(t))^{1 / 2} \\
& \quad \leqslant C_{2} \ln N(\xi(t)+\eta)+C_{3}(\ln N)^{1 / 2} N^{-1 / 2}(\xi(t)+\eta)^{3 / 2} .
\end{aligned}
$$


Здесь мы использовали неравенство Шварца, (3.5), (1.11), (7.1). Константы $C_{2}$ и $C_{3}$ зависят лишь от констант $B_{12}, \mu_{1}^{6}, \mu_{2}^{6}$ и от области $\Omega$. Кроме того, имеет место следующая оценка:

$$
\begin{aligned}
& \left\|w_{x_{i} x_{j}}(x, t)\left(R_{N} Q u_{x_{r} t}\right)(x, t) w_{t t}(x, t)\right\|_{L_{1}(\Omega)} \\
& \quad \leqslant\left\|w_{x_{i} x_{j}}(x, t)\right\|_{L_{\infty}(\Omega)}\left\|\left(R_{N} Q u_{x_{r} t}\right)(x, t)\right\|_{L_{2}(\Omega)}\left\|w_{t t}(x, t)\right\|_{L_{2}(\Omega)} \\
& \quad \leqslant C_{4}(\ln N)^{1 / 2} N^{-1}(\xi(t)+\eta)^{3 / 2}+C_{5} N^{-3 / 2}(\xi(t)+\eta)^{2} .
\end{aligned}
$$

Здесь мы использовали неравенство Шварца, $(4.3),(3.4),(7.1)$. Константы $C_{4}$ и $C_{5}$ зависят лишь от констант $\mu_{1}^{6}, \mu_{2}^{6}$ и от области $\Omega$.

Из (7.8)-(7.10) следует (7.7). Предложение доказано.

\section{§8. Завершение доказательства теоремы 1.1}

Лемма 8.1. Для всех $t \in\left[0, t_{f}\right]$ и всех $r \geqslant 2$ имеет место следующее неравенство:

$$
\begin{aligned}
\frac{d}{d t}(\xi(t)+\eta) \leqslant \mu_{1} & \ln r(\xi(t)+\eta) \\
& +\mu_{2} r^{-1 / 4}(\xi(t)+\eta)^{2}+\left\|Z_{t}(x, t)\right\|_{L_{2}(\Omega)}^{2},
\end{aligned}
$$

где константы $\mu_{1}, \mu_{2}$ зависят лишь от данных задачи (1.1)-(1.9), указанных в формулировке теоремы 1.1, и от области $\Omega$.

ДокаЗАТЕльство (по существу, оно уже проведено). Действительно, рассмотрим неравенство (2.3). Мы оценили все величины (2.4)-(2.17) из правой части (2.3). Эти оценки содержатся соответственно в формулах (2.18)-(2.24), (4.12), (4.20), (5.11), (6.10), (6.15), (7.5), (7.7). Учитывая эти оценки, мы и получим (8.1). Лемма доказана.

Лемма 8.2. Для всех $t_{0}, t \in\left[0, t_{f}\right]$ имеет место следующее неравенство:

$$
\xi(t)+\eta \leqslant \xi\left(t_{0}\right)+\eta+\mu \int_{t_{0}}^{t}(\xi(\tau)+\eta) \ln (\xi(\tau)+\eta) d \tau+\lambda,
$$

где $\mu$ и $\lambda$ - константы, зависящие лишь от данных задачи (1.1)-(1.9), указанных в формулировке теоремы 1.1, и от области $\Omega$.

ДокАЗАТЕЛЬСТво. Положим в (8.1) $r=(\xi(t)+\eta)^{8}, \mu=8 \mu_{1}$. Проинтегрируем (8.1) по $t$ от $t_{0}$ до $t$ и положим $\lambda=\mu_{2} t_{f}+\left\|Z_{t}(x, t)\right\|_{L_{2}\left(\Omega \times\left[0, t_{f}\right]\right)}$, после чего получим неравенство (8.2). Лемма доказана.

Теперь покажем, что решение интегрального неравенства вида (8.1) имеет оценкy. 
Лемма 8.3. Пусть $\theta(t) \in C([0, a])$ u $\theta(t) \geqslant 1$ при $t \in[0, a]$. Пусть функиия $\theta(t)$ для всех $t_{0}, t \in[0, a]$ удовлетворяет следующему неравенству:

$$
\theta(t) \leqslant \theta\left(t_{0}\right)+\varkappa \int_{t_{0}}^{t} \theta(\tau) \ln \theta(\tau) d \tau+\chi
$$

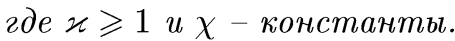

Тогда для всех $t \in[0, a]$

$$
\theta(t) \leqslant K(\varkappa, \chi, a)
$$

где $K(\varkappa, \chi, a)$ - константа, зависящая лишь от $\varkappa, \chi, a$.

ДокАЗАтЕльСтво. Рассматривая (8.3) как неравенство Гронуола, мы получаем следуюшее неравенство для решения неравенства Гронуола (8.3) (см. [8, с. 27]):

$$
\theta(t) \leqslant\left(\chi+\theta\left(t_{0}\right)\right) \exp \left(\varkappa \int_{t_{0}}^{t} \ln \theta(\tau) d \tau\right)
$$

для всех $t \in\left[t_{0}, a\right]$.

Теперь несколько преобразуем правую часть (8.5), получив более удобную для нас оценку. Применяя неравенство Гёльдера, имеем

$$
\begin{aligned}
\theta(t) & \leqslant\left(\chi+\theta\left(t_{0}\right)\right)\left[1+\sum_{n=1}^{\infty} \frac{\varkappa^{n}}{n !}\left(\int_{t_{0}}^{t} \ln \theta(\tau) d \tau\right)^{n}\right] \\
& \leqslant\left(\chi+\theta\left(t_{0}\right)\right)\left[1+\sum_{n=1}^{\infty} \frac{\varkappa^{n}\left(t-t_{0}\right)^{n-1}}{n !} \int_{t_{0}}^{t}(\ln \theta(\tau))^{n} d \tau\right] .
\end{aligned}
$$

Считая, что $t-t_{0} \leqslant \varkappa^{-1}$, из (8.5) получаем

$$
\begin{aligned}
\theta(t) & \leqslant\left(\chi+\theta\left(t_{0}\right)\right)\left[1+\varkappa \sum_{n=1}^{\infty} \frac{1}{n !} \int_{t_{0}}^{t}(\ln \theta(\tau))^{n} d \tau\right] \\
& \leqslant \varkappa\left(\chi+\theta\left(t_{0}\right)\right)\left[1+\sum_{n=1}^{\infty} \frac{1}{n !} \int_{t_{0}}^{t}(\ln \theta(\tau))^{n} d \tau\right] \\
& \leqslant \varkappa\left(\chi+\theta\left(t_{0}\right)\right)\left[1+\int_{t_{0}}^{t} \exp (\ln \theta(\tau)) d \tau\right] \\
& =\varkappa\left(\chi+\theta\left(t_{0}\right)\right)\left(1+\int_{t_{0}}^{t} \theta(\tau) d \tau\right) .
\end{aligned}
$$

Опять применяя оценку решения неравенства Гронуола - теперь уже к (8.7), получаем для $t \in\left[t_{0}, t_{0}+\varkappa^{-1}\right]$

$$
\theta(t) \leqslant \varkappa\left(\chi+\theta\left(t_{0}\right)\right) e^{\chi+\theta\left(t_{0}\right)} .
$$


Введем функцию $\gamma_{\varkappa}, \chi(s)=\varkappa(\chi+s) e^{\chi+s}, s \in[0,+\infty)$, и число $k_{\varkappa, a}$, на единицу большее целой части числа $\varkappa а$. Тогда, используя оценку (8.8), получим для всех $t \in[0, a]$ следуюшую оценку:

$$
\theta(t) \leqslant \gamma_{\varkappa, \chi}^{\left(k_{\varkappa}, a\right)}(\theta(0))
$$

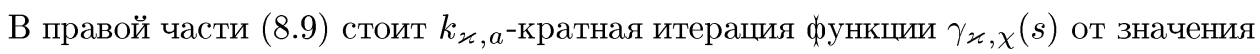
$\theta(0)$. Лемма доказана.

ЗАВЕРШЕНИЕ ДОКАЗАТЕЛЬСТВА ТЕОРЕМЫ 1.1. Оценка (1.18) является непосредственным следствием лемм 8.2 и 8.3 , а (1.19) следует из (4.4) при $p=2$ с учетом (4.1)-(4.3) и (1.18). Оценка (1.20) следует из (6.13) с учетом (1.18). Теорема доказана.

Коснемся теперь вопроса о классе $C_{a}$ гладкости границы $\Gamma$. Граница должна быть такой, чтобы выполнялись неравенства (4.4) и (6.1) (мы неявно использовали аналогичное неравенство для оператора Лапласа). Для этого достаточно, чтобы граница была трижды непрерывно дифференцируема и имела ограниченные производные четвертого порядка.

\section{§9. Доказательство теоремы 2}

Пусть $\chi_{l}(x), l=1,2, \ldots,-$ система собственных функций однородной краевой задачи для оператора $\nabla^{4}$ :

$$
\nabla^{4} \chi_{l}(x)=\lambda_{l} \chi_{l}(x), \quad x \in \Omega,\left.\quad \chi_{l}\right|_{\Gamma}=\left.\frac{\partial \chi_{l}}{\partial n}\right|_{\Gamma}=0
$$

Определим приближения Бубнова-Галёркина

$$
w^{m}(x, t)=\sum_{i=1}^{m} a_{i}^{m}(t) \chi_{i}(x),
$$

где функции времени $a_{i}^{m}(t)$ определяются из системы обыкновенных дифференциальных уравнений

$$
\begin{gathered}
\left(w_{t t}^{m}+\mathcal{D} \nabla^{4} w^{m}-Z-\left(N_{1}^{m} w_{x_{1}}^{m}\right)_{x_{1}}-\left(N_{12}^{m} w_{x_{1}}^{m}\right)_{x_{2}}-\left(N_{2}^{m} w_{x_{2}}^{m}\right)_{x_{2}}\right. \\
\left.-\left(N_{12}^{m} w_{x_{2}}^{m}\right)_{x_{1}}+N_{1}^{m} k_{1}+N_{2}^{m} k_{2}, \chi_{i}\right)_{L_{2}(\Omega)}=0
\end{gathered}
$$

при $i=1, \ldots, m$, с начальньми данными

$$
\begin{aligned}
& a_{i}^{m}(0)=\left(w_{0}, \chi_{i}\right)_{L_{2}(\Omega)}, \\
& \dot{a}_{i}^{m}(0)=\left(w_{1}, \chi_{i}\right)_{L_{2}(\Omega)}
\end{aligned}
$$


при $i=1, \ldots, m$. Функции $u^{m}(x, t)$ и $v^{m}(x, t)$ являются решениями следующей краевой задачи:

$$
\begin{aligned}
\Delta u^{m}+\frac{1+\mu}{1-\mu} \theta_{x_{1}}^{m}= & -\frac{2}{1-\mu}\left[\left(k_{1} w^{m}\right)_{x_{1}}+w_{x_{1}}^{m} w_{x_{1} x_{1}}^{m}+\mu\left(k_{2} w^{m}\right)_{x_{1}}\right. \\
& \left.+\mu w_{x_{1}}^{m} w_{x_{1} x_{2}}^{m}\right]-w_{x_{2}}^{m} w_{x_{1} x_{2}}^{m}-w_{x_{1}}^{m} w_{x_{2} x_{2}}^{m}-X \\
\Delta v^{m}+\frac{1+\mu}{1-\mu} \theta_{x_{2}}^{m}= & -\frac{2}{1-\mu}\left[\left(k_{2} w^{m}\right)_{x_{2}}+w_{x_{2}}^{m} w_{x_{2} x_{2}}^{m}+\mu\left(k_{1} w^{m}\right)_{x_{2}}\right. \\
& \left.+\mu w_{x_{1}}^{m} w_{x_{1} x_{2}}^{m}\right]-w_{x_{1}}^{m} w_{x_{1} x_{2}}^{m}-w_{x_{2}}^{m} w_{x_{1} x_{2}}^{m}-Y
\end{aligned}
$$

с краевыми условиями

$$
\left.u^{m}\right|_{\Gamma}=\left.v^{m}\right|_{\Gamma}=0
$$

И.И. Воровичем в [1] показано, что приближения $w^{m}$ удовлетворяют равномерно по $m$ оценкам вида (1.10)-(1.16). Там же доказано, что можно выбрать подпоследовательность $w^{m_{k}}$, слабо сходящуюся к обобщенному решению $w$ задачи (1.1)-(1.9), в пространстве $H_{3 Q}\left(H_{3 Q}\right.$ в [1] - это пополнение множества бесконечно дифференцируемых на $\Omega \times\left[0, t_{f}\right]$ функций $z(x, t)$, финитных в $\Omega$ при каждом $t \in\left[0, t_{f}\right]$, по норме $\left.\|z\|_{H_{3 Q}}^{2}=\int_{0}^{t_{f}} \int_{\Omega}[\Delta z(x, t)]^{2} d x d t\right)$.

Обобщенное же решение $w$ удовлетворяет следуюшему интегральному соотношению:

$$
\begin{gathered}
\int_{0}^{t_{f}} \int_{\Omega}\left[-w_{t} w^{\prime}+\mathcal{D} \nabla^{2} w \nabla^{2} w^{\prime}+\left(N_{1} k_{1}+N_{2} k_{2}\right) w^{\prime}+\left(N_{1} w_{x_{1}}+N_{12} w_{x_{2}}\right) w_{x_{1}}^{\prime}\right. \\
\left.\quad+\left(N_{12} w_{x_{1}}+N_{2} w_{x_{2}}\right) w_{x_{2}}^{\prime}-Z w^{\prime}\right] d x d t-\int_{\Omega} w_{1} w^{\prime} d x=0
\end{gathered}
$$

для любой функции $w^{\prime} \in D^{0}\left(D^{0}\right.$ в [1] - это пополнение в норме $H_{3 Q}$ множества бесконечно дифференцируемых в $\Omega \times\left[0, t_{f}\right]$ функций $z(x, t)$, финитных в $\Omega$ при каждом $t \in\left[0, t_{f}\right]$ и таких, что $z(x, t) \equiv 0$ для $t_{f}-\delta \leqslant t \leqslant t_{f}$, где $\delta$ - некоторое, определенное для каждой функции число) и начальному условию

$$
\left.\lim _{t \rightarrow 0} \int_{\Omega}\left(w-w_{0}\right)^{2} d x\right|_{t}=0 .
$$

Отметим, что обобщенное решение $w$ удовлетворяет оценкам (1.10)-(1.16) с теми же константами, что и для приближений $w^{m}$. Кроме того, продифференцировав (9.5) по $t$, умножив на $\ddot{a}_{i}^{m}(t)$ и просуммировав по $i$, получим соотношение, из которого для приближений $w^{m}$ следует неравенство (2.3) с величинами (2.4)-(2.17). Это приводит к тому, что все приближения $w^{m}$ удовлетворяют оценкам (1.17)-(1.19), что влечет за собой выполнение оценок (1.20)-(1.22) для обобщенного решения $w$. Отметим, что хотя оценки (4.1)- (4.3) и выполняются для приближений $w^{m}$, доказательство их для приближений непригодно. Поэтому 
здесь мы приведем доказательство оценок вида (4.1)-(4.3) специально для приближений Бубнова-Галёркина.

ПРЕДЛОЖЕНИЕ 9.1. Для всех $m \in \mathbb{Z}^{+} u$ всех $t \in\left[0, t_{f}\right]$ имеют место следующие оченки:

$$
\begin{gathered}
\left\|w^{m}(x, t)\right\|_{H_{2}^{3}(\Omega)} \leqslant \sigma_{23}\left(\xi^{m}(t)+\eta^{m}\right)^{1 / 2}, \\
\left\|w^{m}(x, t)\right\|_{H_{2}^{4}(\Omega)} \leqslant \sigma_{24}\left(\xi^{m}(t)+\eta^{m}\right)^{1 / 2}, \\
\left\|w^{m}(x, t)\right\|_{H_{\infty}^{2}(\Omega)} \leqslant \sigma_{25}\left(\xi^{m}(t)+\eta^{m}\right)^{1 / 2},
\end{gathered}
$$

где константы $\sigma_{23}, \sigma_{24}, \sigma_{25}$ зависят лишь от данных задачи (1.1)-(1.9), указанных в формулировке теоремы 1.1, и от области $\Omega$, но не зависят от $\mathrm{m}$.

ДокАЗАТЕльство. Для любой $\theta(x) \in H_{2}^{1}(\Omega)$ имеет место разложение в ряд:

$$
\theta(x)=\sum_{i=1}^{\infty} b_{i} \chi_{i}(x)
$$

Обозначим через $S_{m}$ ортогональную проекцию $\stackrel{\circ}{H} \underset{2}{2}(\Omega)$ на линейную оболочку $\chi_{1}, \ldots, \chi_{m}$. Умножим теперь $(9.1)$ на $b_{i}$ и просуммируем по $i$ от 1 до $m$. Мы получим следуюшее соотношение:

$$
\begin{aligned}
\mathcal{D}( & \left.\nabla^{4} w^{m}(x, t), \theta(x)\right)_{L_{2}(\Omega)}=-\left(w_{t t}^{m}(x, t), \theta(x)\right)_{L_{2}(\Omega)} \\
& +\left(Z+N_{1}^{m}(x, t) k_{1}(x)+N_{2}^{m}(x, t) k_{2}(x)\right. \\
& +\left(N_{1}^{m}(x, t) w_{x_{1}}^{m}(x, t)\right)_{x_{1}}+\left(N_{12}^{m}(x, t) w_{x_{1}}^{m}(x, t)\right)_{x_{2}} \\
& \left.+\left(N_{2}^{m}(x, t) w_{x_{2}}^{m}(x, t)\right)_{x_{2}}+\left(N_{12}^{m}(x, t) w_{x_{2}}^{m}(x, t)\right)_{x_{1}}, S_{m} \theta(x)\right)_{L_{2}(\Omega)}
\end{aligned}
$$

Интегрируя по частям в $(9.9)$, учитывая ортогональность проекции $S_{m}$, выполнение оценок вида (1.10)-(1.16) для $w^{m}$ равномерно по $m$ и используя неравенство Шварца, получаем

$$
\left(\nabla \Delta w^{m}(x, t), \nabla \theta(x)\right)_{L_{2}(\Omega)} \leqslant \sigma_{23}\left(\xi^{m}(t)+\eta^{m}\right)^{1 / 2}\|\nabla \theta(x)\|_{L_{2}(\Omega)},
$$

откуда в силу произвольности $\theta(x)$ следует (9.6). Теперь, учитывая в (9.9) уже полученную оценку (9.6), а также оценки (6.1) и (9.6) для решений задачи (9.2)-(9.4) и ортогональность $S_{m}$ в $L_{2}(\Omega)$, получим

$$
\left(\nabla^{4} w^{m}(x, t), \theta(x)\right)_{L_{2}(\Omega)} \leqslant \sigma_{24}\left(\xi^{m}(t)+\eta^{m}\right)\|\theta(x)\|_{L_{2}(\Omega)},
$$

откуда в силу произвольности $\theta(x)$ следует (9.7). Оценка (9.8) следует из (9.7). Предложение доказано.

Теперь мы знаем, что обобшенные решения обладают достаточной гладкостью, а следовательно, в силу (9.2) почти всюду удовлетворяют уравнению (1.1), причем $u$ и $v$ находятся из уравнений $(1.4),(1.6)$ с учетом краевых условий (1.7) почти всюду по $t \in\left[0, t_{f}\right]$. Теорема доказана. 


\section{§ 10. Доказательство теоремы 1.3}

Введем следующее обозначение:

$$
\xi^{m, i}(t)=\left\|\frac{\partial^{i+2}}{\partial t^{i+2}} w^{m}(x, t)\right\|_{L_{2}(\Omega)}^{2}+D\left\|\frac{\partial^{i+1}}{\partial t^{i+1}} \Delta w^{m}(x, t)\right\|_{L_{2}(\Omega)}^{2}, \quad i \in \mathbb{Z}^{+} .
$$

В условиях теоремы 1.3 аналогично (2.3) имеет место для всех $m \in \mathbb{Z}^{+}, k_{1}, 1 \leqslant$ $k_{1} \leqslant k \leqslant r+1, t \in\left[0, t_{f}\right]$ следуюшее неравенство:

$$
\xi_{t}^{m, k_{1}}(t) \leqslant \sigma_{26}^{k} \sum_{l=1}^{9} A_{l}^{m, k_{1}}(t),
$$

где константа $\sigma_{26}^{k}$ зависит лишь от коэффициентов задачи (1.1)-(1.9) и от $k$, но не зависит от $m$.

Далее, аналогично (2.4)-(2.17) имеем

$$
\begin{aligned}
& A_{1}^{m, k_{1}}(t)=\left(\frac{\partial^{k_{1}+1}}{\partial t^{k_{1}+1}} Z(x, t), \frac{\partial^{k_{1}+2}}{\partial t^{k_{1}+2}} w^{m}(x, t)\right)_{L_{2}(\Omega)}, \\
& A_{2}^{m, k_{1}}(t)=\sum_{i=1}^{2} \sum_{p_{1}+p_{2}=k_{1}+1} \| \frac{\partial^{p_{1}}}{\partial t^{p_{1}}} w^{m}(x, t) \frac{\partial^{p_{2}}}{\partial t^{p_{2}}} w_{x_{i}}^{m}(x, t) \\
& \times \frac{\partial^{k_{1}+2}}{\partial t^{k_{1}+2}} w^{m}(x, t) \|_{L_{1}(\Omega)}, \\
& A_{3}^{m, k_{1}}(t)=\sum_{i, j=1}^{2} \sum_{p_{1}+p_{2}=k_{1}+1} \| \frac{\partial^{p_{1}}}{\partial t^{p_{1}}} w^{m}(x, t) \frac{\partial^{p_{2}}}{\partial t^{p_{2}}} w_{x_{j}}^{m}(x, t) \\
& \times \frac{\partial^{k_{1}+2}}{\partial t^{k_{1}+2}} w^{m}(x, t) \|_{L_{1}(\Omega)}, \\
& A_{4}^{m, k_{1}}(t)=\sum_{i, j=1}^{2} \sum_{p_{1}+p_{2}=k_{1}+1} \| \frac{\partial^{p_{1}}}{\partial t^{p_{1}}} w^{m}(x, t) \frac{\partial^{p_{2}}}{\partial t^{p_{2}}} w_{x_{i} x_{j}}^{m}(x, t) \\
& \times \frac{\partial^{k_{1}+2}}{\partial t^{k_{1}+2}} w^{m}(x, t) \|_{L_{1}(\Omega)}, \\
& A_{5}^{m, k_{1}}(t)=\left\|\frac{\partial^{k_{1}+1}}{\partial t^{k_{1}+1}} w^{m}(x, t) \frac{\partial^{k_{1}+2}}{\partial t^{k_{1}+2}} w^{m}(x, t)\right\|_{L_{1}(\Omega)}, \\
& A_{6}^{m, k_{1}}(t)=\sum_{i, j, r, s=1}^{2} \sum_{p_{1}+p_{2}+p_{3}=k_{1}+1} \| \frac{\partial^{p_{1}}}{\partial t^{p_{1}}} w_{x_{i}}^{m}(x, t) \frac{\partial^{p_{2}}}{\partial t^{p_{2}}} w_{x_{j}}^{m}(x, t) \\
& \times \frac{\partial^{p_{3}}}{\partial t^{p_{3}}} w_{x_{r} x_{s}}^{m}(x, t) \frac{\partial^{k_{1}+2}}{\partial t^{k_{1}+2}} w^{m}(x, t) \|_{L_{1}(\Omega)},
\end{aligned}
$$




$$
\begin{gathered}
A_{7}^{m, k_{1}}(t)=\sum_{i=1}^{2}\left(\left\|\frac{\partial^{k_{1}+1}}{\partial t^{k_{1}+1}} u_{x_{i}}^{m}(x, t) \frac{\partial^{k_{1}+2}}{\partial t^{k_{1}+2}} w^{m}(x, t)\right\|_{L_{1}(\Omega)}\right. \\
\left.+\left\|\frac{\partial^{k_{1}+1}}{\partial t^{k_{1}+1}} v_{x_{i}}^{m}(x, t) \frac{\partial^{k_{1}+2}}{\partial t^{k_{1}+2}} w^{m}(x, t)\right\|_{L_{1}(\Omega)}\right), \\
A_{8}^{m, k_{1}}(t)=\sum_{i, j, r=1}^{2} \sum_{p_{1}+p_{2}=k_{1}+1}\left(\left\|\frac{\partial^{p_{1}}}{\partial t^{p_{1}}} w_{x_{i}}^{m}(x, t) \frac{\partial^{p_{2}}}{\partial t^{p_{2}}} u_{x_{j} x_{r}}^{m}(x, t) \frac{\partial^{k_{1}+2}}{\partial t^{k_{1}+2}} w^{m}(x, t)\right\|_{L_{1}(\Omega)}\right. \\
\left.+\left\|\frac{\partial^{p_{1}}}{\partial t^{p_{1}}} w_{x_{i}}^{m}(x, t) \frac{\partial^{p_{2}}}{\partial t^{p_{2}}} v_{x_{j} x_{r}}^{m}(x, t) \frac{\partial^{k_{1}+2}}{\partial t^{k_{1}+2}} w^{m}(x, t)\right\|_{L_{1}(\Omega)}\right), \\
A_{9}^{m, k_{1}}(t)=\sum_{i, j, r=1}^{2} \sum_{p_{1}+p_{2}=k_{1}+1}\left(\left\|\frac{\partial^{p_{1}}}{\partial t^{p_{1}}} u_{x_{i}}^{m}(x, t) \frac{\partial^{p_{2}}}{\partial t^{p_{2}}} w_{x_{j} x_{r}}^{m}(x, t) \frac{\partial^{k_{1}+2}}{\partial t^{k_{1}+2}} w^{m}(x, t)\right\|_{L_{1}(\Omega)}\right. \\
\left.+\left\|\frac{\partial^{p_{1}}}{\partial t^{p_{1}}} v_{x_{i}}^{m}(x, t) \frac{\partial^{p_{2}}}{\partial t^{p_{2}}} w_{x_{j} x_{r}}^{m}(x, t) \frac{\partial^{k_{1}+2}}{\partial t^{k_{1}+2}} w^{m}(x, t)\right\|_{L_{1}(\Omega)}\right) .
\end{gathered}
$$

Оценки величин $A_{i}^{m, k}(t), i=1, \ldots, 9$, не столь трудоемки, как оценки аналогичных величин $A_{i}(t), i=1, \ldots, 14$, поскольку мы уже располагаем сильными оценками вида (1.17)-(1.19).

ПРЕДЛОЖЕНИЕ 10.1. В условиях теоремы 1.3 для всех $m \in \mathbb{Z}^{+}, k_{1}, \quad 1 \leqslant$ $k_{1} \leqslant k, t \in\left[0, t_{f}\right]$ имеет место следующая оценка:

$$
\left\|\frac{\partial^{k_{1}}}{\partial t^{k_{1}}} w^{m}(x, t)\right\|_{H_{2}^{4}(\Omega)} \leqslant \sigma_{27}^{k}\left[\left(\xi^{m, k}(t)\right)^{1 / 2}+\left(\sum_{i=1}^{k_{1}-1}\left\|\frac{\partial^{i}}{\partial t^{i}} w^{m}(x, t)\right\|_{H_{2}^{4}(\Omega)}\right)^{3}+1\right],
$$

где константа $\sigma_{27}^{k}$ зависит лищь от данных начально-краевой задачи (1.1)-(1.9), указанных в формулировке теоремь 1.3, от области $\Omega$ и числа $k$ ине зависит от $m$.

ДокАЗАТЕЛЬСТво. Продифференцируем уравнения (9.1) $k_{1}$ раз по $t$. Из полученной таким образом системы уравнений для всех $m \in \mathbb{Z}^{+}$и $t \in\left[0, t_{f}\right]$ следует неравенство

$$
\begin{gathered}
\left\|\frac{\partial^{k_{1}}}{\partial t^{k_{1}}} w^{m}(x, t)\right\|_{H_{2}^{4}(\Omega)} \leqslant C_{1}\left(\left\|\frac{\partial^{k_{1}}}{\partial t^{k_{1}}} Z(x, t)\right\|_{L_{2}(\Omega)}\right. \\
+\sum_{i=1}^{2} \sum_{p_{1}+p_{2}=k_{1}}\left\|\frac{\partial^{p_{1}}}{\partial t^{p_{1}}} w^{m}(x, t) \frac{\partial^{p_{2}}}{\partial t^{p_{2}}} w_{x_{i}}^{m}(x, t)\right\|_{L_{2}(\Omega)} \\
+\sum_{i, j=1}^{2}\left\|\frac{\partial^{p_{1}}}{\partial t^{p_{1}}} w_{x_{i}}^{m}(x, t) \frac{\partial^{p_{2}}}{\partial t^{p_{2}}} w_{x_{j}}^{m}(x, t)\right\|_{L_{2}(\Omega)}
\end{gathered}
$$




$$
\begin{aligned}
& +\sum_{i, j=1}^{2} \sum_{p_{1}+p_{2}=k_{1}}\left\|\frac{\partial^{p_{1}}}{\partial t^{p_{1}}} w^{m}(x, t) \frac{\partial^{p_{2}}}{\partial t^{p_{2}}} w_{x_{i} x_{j}}^{m}(x, t)\right\|_{L_{2}(\Omega)}+\left\|\frac{\partial^{k_{1}}}{\partial t^{k_{1}}} w^{m}(x, t)\right\|_{L_{2}(\Omega)} \\
& +\sum_{i, j, r, s=1}^{2}\left\|\frac{\partial^{p_{1}}}{\partial t^{p_{1}}} w_{x_{i}}^{m}(x, t) \frac{\partial^{p_{2}}}{\partial t^{p_{2}}} w_{x_{j}}^{m}(x, t) \frac{\partial^{p_{3}}}{\partial t^{p_{3}}} w_{x_{r} x_{s}}^{m}(x, t)\right\|_{L_{2}(\Omega)} \\
& +\sum_{i=1}^{2}\left(\left\|\frac{\partial^{k_{1}}}{\partial t^{k_{1}}} u_{x_{i}}^{m}(x, t)\right\|_{L_{2}(\Omega)}+\left\|\frac{\partial^{k_{1}}}{\partial t^{k_{1}}} v_{x_{i}}^{m}(x, t)\right\|_{L_{2}(\Omega)}\right) \\
& +\sum_{i, j, r=1}^{2} \sum_{p_{1}+p_{2}=k_{1}}\left(\left\|\frac{\partial^{p_{1}}}{\partial t^{p_{1}}} w_{x_{i}}^{m}(x, t) \frac{\partial^{p_{2}}}{\partial t^{p_{2}}} u_{x_{j} x_{r}}^{m}(x, t)\right\|_{L_{2}(\Omega)}\right. \\
& +\left\|\frac{\partial^{p_{1}}}{\partial t^{p_{1}}} w_{x_{i}}^{m}(x, t) \frac{\partial^{p_{2}}}{\partial t^{p_{2}}} v_{x_{j} x_{r}}^{m}(x, t)\right\|_{L_{2}(\Omega)} \\
& +\left\|\frac{\partial^{p_{1}}}{\partial t^{p_{1}}} u_{x_{i}}^{m}(x, t) \frac{\partial^{p_{2}}}{\partial t^{p_{2}}} w_{x_{j} x_{r}}^{m}(x, t)\right\|_{L_{2}(\Omega)} \\
& \left.\left.+\left\|\frac{\partial^{p_{1}}}{\partial t^{p_{1}}} v_{x_{i}}^{m}(x, t) \frac{\partial^{p_{2}}}{\partial t^{p_{2}}} w_{x_{j} x_{r}}^{m}(x, t)\right\|_{L_{2}(\Omega)}\right)\right)
\end{aligned}
$$

где константа $C_{1}$ зависит лишь от коэффициентов уравнения (1.1).

Учитывая неравенства коэрцитивности вида (6.1), вид правых частей уравнений (9.2), (9.3), оценки приближений $w^{m}$ вида (1.17)-(1.19) и теорему вложения $H_{2}^{2}(\Omega)$ в $L_{\infty}(\Omega)$ при $p \leqslant k_{1}-1$, получаем

$$
\begin{aligned}
& \left\|\frac{\partial^{p}}{\partial t^{p}} u^{m}(x, t)\right\|_{H_{2}^{2}(\Omega)}+\left\|\frac{\partial^{p}}{\partial t^{p}} v^{m}(x, t)\right\|_{H_{2}^{2}(\Omega)} \\
& \quad \leqslant C_{2}\left[\left(\sum_{i=1}^{p}\left\|\frac{\partial^{i}}{\partial t^{i}} w^{m}(x, t)\right\|_{H_{2}^{4}(\Omega)}\right)^{2}+1\right]
\end{aligned}
$$

где константа $C_{2}$ зависит лишш от данных задачи (1.1)-(1.9), указанных в формулировке теоремы 1.3 , от $k$ и от области $\Omega$.

Аналогично имеем

$$
\begin{aligned}
& \left\|\frac{\partial^{k_{1}}}{\partial t^{k_{1}}} u^{m}(x, t)\right\|_{H_{2}^{2}(\Omega)}+\left\|\frac{\partial^{k_{1}}}{\partial t^{k_{1}}} v^{m}(x, t)\right\|_{H_{2}^{2}(\Omega)} \\
& \quad \leqslant C_{3}\left[\left(\xi^{m, k_{1}}(t)\right)^{1 / 2}+\left(\sum_{i=1}^{k_{1}-1}\left\|\frac{\partial^{i}}{\partial t^{i}} w^{m}(x, t)\right\|_{H_{2}^{4}(\Omega)}\right)^{2}+1\right]
\end{aligned}
$$

где $C_{3}$ зависит от тех же величин, что и $C_{2}$.

Представим правую часть (10.12) в виде суммы двух слагаемых, $Q_{1}(t)$ и $Q_{2}(t)$, где $Q_{1}(t)$ состоит из величин, содержащих частную производную, в которой дифференцирование по $t$ имеет порядок $k_{1}, Q_{2}$ состоит из остальных слагаемых. 
Оценим сначала $Q_{1}(t)$, используя теорему вложения $H_{2}^{2}(\Omega)$ в $L_{\infty}(\Omega)$ и равномерные оценки приближений $w^{m}$ вида (1.17)-(1.19). Имеем

$$
Q_{1}(t) \leqslant C_{4}\left[\left(\xi^{m, k_{1}}(t)\right)^{1 / 2}+\left(\sum_{i=1}^{k_{1}-1}\left\|\frac{\partial^{i}}{\partial t^{i}} w^{m}(x, t)\right\|_{H_{2}^{4}(\Omega)}\right)^{2}+1\right] .
$$

Аналогично оценим $Q_{2}(t)$. Получаем

$$
Q_{2}(t) \leqslant C_{5}\left[\left(\sum_{i=1}^{k_{1}-1}\left\|\frac{\partial^{i}}{\partial t^{i}} w^{m}(x, t)\right\|_{H_{2}^{4}(\Omega)}\right)^{3}+1\right] .
$$

Константы $C_{4}$ и $C_{5}$ зависят от тех же величин, что и константа $C_{2}$. Оценки $(10.12)$, (10.15) и (10.16) дают (10.11). Предложение доказано.

ПРЕДЛОЖЕНИЕ 10.2. В условиях теоремы 1.3 для всех $m \in \mathbb{Z}^{+}, k_{1}, 1 \leqslant$ $k_{1} \leqslant k, \quad t \in\left[0, t_{f}\right]$ имеет место следующая оценка:

$$
\left\|\frac{\partial^{k_{1}}}{\partial t^{k_{1}}} w^{m}(x, t)\right\|_{H_{2}^{4}(\Omega)} \leqslant \sigma_{28}^{k, k_{1}}\left[\left(\xi^{m, k_{1}}(t)\right)^{1 / 2}+\left(\sum_{i=1}^{k_{1}-1} \xi^{m, i}(t)\right)^{3}+1\right],
$$

где константа $\sigma_{28}^{k, k_{1}}$ зависит лишь от данных задачи (1.1)-(1.9), указанных в формулировке теоремь 1.3 , от области $\Omega$, от чисел $k, k_{1}$ и не зависит от $m$.

ДоКАЗАТЕЛЬСТво (проведем его по индукции по $k_{1}$ ). Для $k_{1}=0$ у нас есть оценка (1.19). Опираясь на неравенство (10.11) и используя индуктивное предположение о выполнении (10.17) при $k_{1}$, покажем, что (10.17) выполняется при $k_{1}+1$. Имеем

$$
\begin{aligned}
& \left\|\frac{\partial^{k_{1}+1}}{\partial t^{k_{1}+1}} w^{m}(x, t)\right\|_{H_{2}^{4}(\Omega)} \leqslant \sigma_{27}^{k}\left[\left(\xi^{m, k_{1}+1}(t)\right)^{1 / 2}+\left(\sum_{i=1}^{k_{1}}\left\|\frac{\partial^{i}}{\partial t^{i}} w^{m}(x, t)\right\|_{H_{2}^{4}(\Omega)}\right)^{3}+1\right] \\
& \leqslant \sigma_{25}^{k}\left[\left(\xi^{m, k_{1}+1}(t)\right)^{1 / 2}+\left(\sum_{i=1}^{k_{1}} \sigma_{28}^{k, i}\left(\left(\xi^{m, i}(t)\right)^{1 / 2}+\left(\sum_{j=1}^{i-1} \xi^{m, j}(t)\right)^{3}\right)^{3}+1\right)\right] \\
& \leqslant \sigma_{27}^{k}\left(\xi^{m, k_{1}+1}(t)\right)^{1 / 2}+C_{1}\left[\left(\sum_{i=1}^{k_{1}} \xi^{m, i}(t)\right)^{3}+1\right]
\end{aligned}
$$

где константа $C_{1}$ зависит лишь от констант $\sigma_{27}^{k}, \sigma_{28}^{k, i}, i=1, \ldots, k_{1}$, и $k_{1}$. Тем самым предложение доказано.

ПреДлОЖенИЕ 10.3. В условиях теоремы 1.3 для всех $m \in \mathbb{Z}^{+}, k_{1}, 1 \leqslant$ $k_{1} \leqslant k, t \in\left[0, t_{f}\right]$

$$
\xi_{t}^{m, k_{1}}(t) \leqslant \sigma_{29}^{k, k_{1}}\left[\xi^{m, k_{1}}(t)+\left(\sum_{i=1}^{k_{1}-1} \xi^{m, i}(t)\right)^{3^{k_{1}+2}}+1\right],
$$

где константа $\sigma_{29}^{k, k_{1}}$ зависит лишь от данных начально-краевой задачи (1.1)-(1.9), указанных в формулировке теоремы 1.3, от области $\Omega$ и чисел $k, k_{1}$ и не зависит от $m$. 
ДокАЗАТЕЛЬСтво. Представим правую часть (10.1) в виде суммы двух слагаемых, $Q^{1}(t)$ и $Q^{2}(t)$, где $Q^{1}(t)$ состоит из величин, содержаших частную производную, в которой дифференцирование по $t$ имеет порядок $k_{1}+1$, а $Q^{2}(t)$ состоит из остальных слагаемых. С учетом (10.2)-(10.10) и оценки $(10.17) Q^{1}(t)$ оценивается аналогично (10.15). Сначала, используя ограниченность вложения $H_{2}^{2}(\Omega)$ в $L_{\infty}(\Omega)$, оценки $(1.17)-(1.19)$, вид $\xi^{m, i}(t)$ и $(10.17)$, имеем

$$
\begin{gathered}
\left\|\frac{\partial^{k_{1}+1}}{\partial t^{k_{1}+1}} u^{m}(x, t)\right\|_{H_{2}^{2}(\Omega)}+\left\|\frac{\partial^{k_{1}+1}}{\partial t^{k_{1}+1}} v^{m}(x, t)\right\|_{H_{2}^{2}(\Omega)} \\
\leq C_{1}\left[\left(\xi^{m, k_{1}}(t)\right)^{1 / 2}+\left\|\frac{\partial^{k_{1}}}{\partial t^{k_{1}}} w^{m}(x, t)\right\|_{H_{2}^{2}(\Omega)}\left\|\frac{\partial}{\partial t} w^{m}(x, t)\right\|_{H_{2}^{4}(\Omega)}\right. \\
\left.+\sum_{i=1}^{k_{1}-1}\left\|\frac{\partial^{i}}{\partial t^{i}} w^{m}(x, t)\right\|_{H_{2}^{4}(\Omega)}^{2}+1\right] \\
\leq C_{2}\left[\left(\xi^{m, k_{1}}(t)\right)^{1 / 2}+\left(\sum_{i=1}^{k_{1}-1} \xi^{m, i}(t)\right)^{3^{k_{1}+1}}+1\right],
\end{gathered}
$$

где константа $C_{2}$ зависит от тех же величин, что и $\sigma_{29}^{k, k_{1}}$.

Теперь, используя те же результаты, неравенства Шварца и Юнга, а также (10.19), оценим $Q^{1}(t)$. Получаем

$$
Q^{1}(t) \leqslant C_{3}\left[\xi^{m, k_{1}}(t)+\left(\sum_{i=1}^{k_{1}-1} \xi^{m, i}(t)\right)^{3^{k_{1}+2}}+1\right]
$$

где константа $C_{3}$ зависит от тех же величин, что и $C_{2}$.

Теперь сходным образом оценим $Q^{2}(t)$, учитывая (10.19) для производных по $t$ меньшего порядка и используя (10.13). Имеем

$$
\begin{aligned}
Q^{2}(t) \leqslant & C_{4}\left[\left(\left\|\frac{\partial^{k_{1}}}{\partial t^{k_{1}}} w^{m}(x, t)\right\|_{H_{2}^{2}(\Omega)}^{2}+\left\|\frac{\partial^{k_{1}}}{\partial t^{k_{1}}} u^{m}(x, t)\right\|_{H_{2}^{2}(\Omega)}^{2}\right.\right. \\
& \left.+\left\|\frac{\partial^{k_{1}}}{\partial t^{k_{1}}} v^{m}(x, t)\right\|_{H_{2}^{2}(\Omega)}^{2}\right)\left(\left\|\frac{\partial}{\partial t} w^{m}(x, t)\right\|_{H_{2}^{4}(\Omega)}^{2}+1\right) \\
& \left.+\left(\sum_{i=1}^{k_{1}-1} \xi^{m, i}(t)\right)^{3^{k_{1}+2}}+1\right] \leqslant C_{5}\left[\left(\sum_{i=1}^{k_{1}-1} \xi^{m, i}(t)\right)^{3^{k_{1}+2}}+1\right] .
\end{aligned}
$$

Константа $C_{5}$ зависит от тех же величин, что и $C_{3}$. Оценки $(10.20)$ и $(10.21)$ дают (10.18). Предложение доказано. 
ПРЕДЛОЖЕНИЕ 10.4. В условиях теоремы 1.3 для всех $m \in \mathbb{Z}^{+}, t \in\left[0, t_{f}\right]$ имеют место следующие оценки:

$$
\begin{gathered}
\max _{t \in\left[0, t_{f}\right]}\left(\left\|\frac{\partial^{r+2}}{\partial t^{r+2}} w^{m}(x, t)\right\|_{L_{2}(\Omega)}+\left\|\frac{\partial^{r+1}}{\partial t^{r+1}} \Delta w^{m}(x, t)\right\|_{L_{2}(\Omega)}\right) \leqslant K_{4}^{r}, \\
\max _{t \in\left[0, t_{f}\right]}\left\|\frac{\partial^{r}}{\partial t^{r}} \nabla^{4} w^{m}(x, t)\right\|_{L_{2}(\Omega)} \leqslant K_{5}^{r} \\
\max _{t \in\left[0, t_{f}\right]}\left(\left\|\frac{\partial^{r}}{\partial t^{r}} u^{m}(x, t)\right\|_{H_{2}^{4}(\Omega)}+\left\|\frac{\partial^{r}}{\partial t^{r}} v^{m}(x, t)\right\|_{H_{2}^{4}(\Omega)}\right) \leqslant K_{6}^{r}
\end{gathered}
$$

где константы $K_{4}^{r}, K_{5}^{r}, K_{6}^{r}$ зависят лишь от величин, указанных в формулировке теоремы 1.3.

ДокАЗАТЕльство. Применяя оценку решения неравенства Гронуола и (10.18), мы получим рекуррентную оценку

$$
\xi^{m, k_{1}}(t) \leqslant\left[\xi^{m, k_{1}}(0)+\sigma_{29}^{k, k_{1}}\left(\int_{0}^{t}\left(\sum_{i=1}^{k_{1}-1} \xi^{m, i}(\tau)\right)^{3^{k_{1}+2}} d \tau+t_{f}\right)\right] \exp \left(\sigma_{29}^{k, k_{1}} t_{f}\right) .
$$

Из (10.25) шаг за шагом получим (10.22). Оценка (10.23) получится из (10.17) и (10.22), a (10.24) получится из (10.14) и (10.22). Предложение доказано.

ПРЕДЛОЖЕНИЕ 10.5. В условиях теоремы 1.3 для решения $w(x, t)$ начально-краевой задачи (1.1)-(1.9) для всех $t \in\left[0, t_{f}\right]$ имеют место следующие оченки:

$$
\begin{aligned}
& \underset{t \in\left[0, t_{f}\right]}{\operatorname{vraimax} \max }\left(\left\|\frac{\partial^{r+2}}{\partial t^{r+2}} w(x, t)\right\|_{L_{2}(\Omega)}+\left\|\frac{\partial^{r+1}}{\partial t^{r+1}} \Delta w(x, t)\right\|_{L_{2}(\Omega)}\right) \leqslant K_{4}^{r}, \\
& \underset{t \in\left[0, t_{f}\right]}{\operatorname{vraimax}}\left\|\frac{\partial^{r}}{\partial t^{r}} \nabla^{4} w(x, t)\right\|_{L_{2}(\Omega)} \leqslant K_{5}^{r}, \\
& \underset{t \in\left[0, t_{f}\right]}{\operatorname{vraimax}}\left(\left\|\frac{\partial^{r}}{\partial t^{r}} u(x, t)\right\|_{H_{2}^{4}(\Omega)}+\left\|\frac{\partial^{3}}{\partial t^{r}} v(x, t)\right\|_{H_{2}^{4}(\Omega)}\right) \leqslant K_{6}^{r} .
\end{aligned}
$$

ДокАЗАТЕЛЬСТво. Перейдя к пределу по подпоследовательности приближений, сходящейся к решению задачи (1.1)-(1.9), из оценок (10.22)-(10.24) получим оценки (10.26)-(10.28). Предложение доказано.

ОКОНЧАНИЕ ДОКАЗАТЕЛЬСТВА ТЕОРЕМЫ 1.3. Нам осталось доказать две серии оценок (1.23) и (1.24). Мы уже доказали первые три оценки из первой серии. Это оценки (10.22) и (10.23). Доказана также оценка (10.24), первая из серии (1.24). Остальные оценки последовательно получаются из уравнения (1.1) и задачи $(1.4),(1.5),(1.7)$ с учетом неравенств коэрцитивности вида (6.1) и предыдущих оценок каждой серии, а также оценок (1.17)-(1.19) и неравенств коэрцитивности для операторов $\Delta$ и $\Delta^{2}$ с однородными условиями, неравенств Шварца и Гёльдера и теорем вложения. Теорема доказана. 
Класс $S_{a}^{r}$ гладкости границ $Г$ определяется условиями выполнения неравенств коэрцитивности для тех же задач, что и класс $S_{a}$, только соответственно для производных более высокого порядка.

Автор выражает благодарность В.И. Юдовичу и И.И. Воровичу за полезное обсуждение работы.

\section{Список литературы}

1. Ворович И. И. О некоторых прямых методах в нелинейной теории колебаний пологих оболочек // Изв. АН СССР. Сер. матем. 1957. Т. 21. №6. С. 747-784.

2. Седенко В.И. Единственность обобщенного решения начально-краевой задачи нелинейной теории колебаний пологих оболочек // Докл. АН СССР. 1991. Т. 316. №6. C. 1319-1322.

3. Седенко В. И. Теорема единственности обобщенного решения начально-краевой задачи нелинейной теории колебаний пологих оболочек с малой инерцией продольных перемещений // Изв. АН СССР. Сер. мех. тв. тела. 1991. №6. С. 142-150.

4. Морозов Н. . О нелинейных колебаниях тонких пластин с учетом инерции вращения // Докл. АН СССР. 1967. Т. 176. № 3. С. 522-525.

5. Бесов О.В., Ильин В. П., Никольский С. М. Интегральные представления функций и теоремы вложения. М.: Наука, 1975.

6. Стейн И. Сингулярные интегралы и дифференциальные свойства функций. М.: Мир, 1973.

7. Ладыженская $О . А$. Уральцева $H$. . Линейные и квазилинейные уравнения эллиптического типа. М.: Наука, 1973.

8. Хартман . Обыкновенные дифференциальные уравнения. М.: Мир, 1970.

Ростов-на-Дону

Поступило в редакцию

14.VI.1994 